\title{
Review of optical coherence tomography in oncology
}

\author{
Jianfeng Wang \\ Yang $\mathrm{Xu}$ \\ Stephen A. Boppart
}




\title{
Review of optical coherence tomography in oncology
}

\author{
Jianfeng Wang, ${ }^{a}$ Yang $\mathrm{Xu}{ }^{a, b}$ and Stephen A. Boppart ${ }^{a, b, c, d, *}$ \\ aUniversity of Illinois at Urbana-Champaign, Beckman Institute for Advanced Science and Technology, Urbana, Illinois, United States \\ bUniversity of Illinois at Urbana-Champaign, Department of Electrical and Computer Engineering, Urbana, Illinois, United States \\ 'University of Illinois at Urbana-Champaign, Department of Bioengineering, Urbana, Illinois, United States \\ dUniversity of Illinois at Urbana-Champaign, Carle-Illinois College of Medicine, Urbana, Illinois, United States
}

\begin{abstract}
The application of optical coherence tomography (OCT) in the field of oncology has been prospering over the past decade. OCT imaging has been used to image a broad spectrum of malignancies, including those arising in the breast, brain, bladder, the gastrointestinal, respiratory, and reproductive tracts, the skin, and oral cavity, among others. OCT imaging has initially been applied for guiding biopsies, for intraoperatively evaluating tumor margins and lymph nodes, and for the early detection of small lesions that would often not be visible on gross examination, tasks that align well with the clinical emphasis on early detection and intervention. Recently, OCT imaging has been explored for imaging tumor cells and their dynamics, and for the monitoring of tumor responses to treatments. This paper reviews the evolution of OCT technologies for the clinical application of OCT in surgical and noninvasive interventional oncology procedures and concludes with a discussion of the future directions for OCT technologies, with particular emphasis on their applications in oncology. ๑ 2017 Society of PhotoOptical Instrumentation Engineers (SPIE) [DOI: 10.1117/1.JBO.22.12.121711]
\end{abstract}

Keywords: optical coherence tomography; oncology; cancer; tumor; intraoperative; surgery.

Paper 170658SSVR received Oct. 9, 2017; accepted for publication Dec. 4, 2017; published online Dec. $22,2017$.

\section{Introduction}

In the field of oncology, imaging has been playing a fundamental role, providing valuable morphological, anatomical, metabolic, and molecular information for more-sophisticated treatments, and therefore better outcomes for patients diagnosed with cancer. ${ }^{1,2}$ Imaging has been utilized in virtually every management step, including the initial screening, staging, restaging, and posttherapeutic follow-up of a wide range of cancers. ${ }^{1,2}$ Different imaging modalities have been exploited to aid clinicians in the diagnosis, staging, and treatment of human cancers, which include the established oncological imaging modalities (CT, computed tomography; MRI, magnetic resonance imaging; single-photon emission computed tomography; positron emission tomography; and ultrasound imaging), ${ }^{3}$ as well as the more recently developed optical imaging methods [i.e., optical coherence tomography (OCT) and diffuse optical tomography]. ${ }^{3-5}$ Established oncological imaging modalities have been contributing to better human cancer management, but the spatial resolutions and the soft-tissue contrast have not always met expectations, ${ }^{1-3}$ and unsurprisingly, cancer patients are often being imaged with ionizing radiation, albeit at doses as low as possible. ${ }^{1-3}$ Compared with the established oncological imaging modalities characterized by large fields of view and deep imaging penetration depths, optical imaging methods generally sacrifice these metrics for other performance advantages. The advantages of optical imaging methods are multifold: first, optical imaging methods reduce patient exposure to harmful radiation using nonionizing radiation (i.e., visible and near-infrared light sources); second, optical imaging offers high resolution (on the order of micrometers and submicrometers) and has always

*Address all correspondence to: Stephen A. Boppart, E-mail: boppart@illinois .edu been used in pathology to assess biopsies and tissue specimens; and third, optical imaging is particularly useful for visualizing soft tissues with a large degree of contrast mechanisms to help in differentiating cell and tissue types, as well as disease. ${ }^{6}$

Among the various optical modalities developed for oncology imaging, OCT, an established imaging technique based on the principle of low coherence interferometry, has stood out with a variety of oncological applications both at the laboratory bench and in the clinics. ${ }^{7-16}$ Fundamentally, the contrast mechanism of OCT relies on the variations of refractive index and the light scattering properties associated with different tissue structures. The technique was first introduced by its name in 1991 for the investigation of the human eye by a Massachusetts Institute of Technology (MIT) team headed by Huang et al. ${ }^{7}$ It initially proved to be of significant value in the imaging of transparent, weakly scattering media (i.e., anterior and posterior of the human eye), and gained its largest clinical impact in the ophthalmology community. ${ }^{17,18}$ In contrast to the eye, nontransparent tissues (i.e., breast, skin, etc.) exhibit a high degree of optical scattering and absorption, resulting in a limited OCT imaging depth. ${ }^{19}$ When illuminating nontransparent tissue, most of the incident photons are scattered more than once, leading primarily to the coherent rejection of these multiple scattered photons but also to artifacts in the OCT images. ${ }^{19}$ In addition, OCT suffers from the trade-off between transverse resolution and depth of field. ${ }^{20}$ However, advances made in the past decade in OCT technology have it made it possible to image nontransparent tissues with high spatial resolution at large (up to 1 to $2 \mathrm{~mm}$ ) imaging depth, depending on the optical properties of the tissue, enabling OCT to be applied in a wide range of other clinical and research applications, including the field of oncology, ${ }^{7-16}$ where there remains a clear and unmet clinical need to effectively detect and diagnose cancer, as well as monitor the treatment response of cancer. Typically, effective cancer treatment 
depends on early detection and for most solid tumors, successful surgical removal. Usually, cancer diagnoses occur through histopathology and established oncological imaging modalities. Histopathology requires tissue biopsy followed by labor- and time-intensive tissue sample preparation and microscopic examination of the histological slides.

There are several scenarios where OCT has been contributing tremendously for the early detection of cancer: (I) OCT can be used for cancer screening at higher resolutions than currently possible with the more established oncological imaging modalities, albeit with the challenge of gaining optical access to the tissue site; (II) OCT can be used for guided biopsy, which is especially useful in situations where conventional biopsy is not effective, or where biopsies are associated with multiple complications; (III) OCT can aid in the intraoperative imaging of cancer in the operation room by providing real-time feedback to the surgeons; (IV) OCT can be used for the monitoring of tumor responses to treatments [i.e., photodynamic therapy, radiotherapy, ablative (thermal- or cryo-) therapy, and chemotherapy etc.]. In this review, the development and widespread demonstrations of OCT in oncology will be presented with an emphasis on ex vivo and in vivo cancer diagnosis applications. Potential improvements and future directions of OCT techniques, and their successful translation into the field of oncology, will also be discussed.

\section{Cancer Detection and Diagnosis}

The current gold standard for cancer detection is based on biopsied tissues followed by histological analysis. Histopathology, while reliable, does not allow surgeons to differentiate neoplastic lesions from normal tissues in real time or in vivo, which would be extremely beneficial for the complete removal of a tumor. Unlike normal tissue with well-organized tissue structure, the structure of cancer tissue is often disorganized and typically characterized by variable cell sizes, abnormal shapes, and enlarged nuclei, resulting in different optical scattering properties, and enabling OCT to reveal differences between normal and cancerous tissues with high spatial resolution. Compared with established oncological imaging modalities, the high soft-tissue contrast offered by OCT (based on refractive index differences) facilitates detailed interpretation of soft-tissue anatomy, which is crucial for the early cancer diagnosis. In addition, OCT can be integrated into small probes and catheters, making it suitable to access internal organs for cancer imaging and diagnosis. Compared with other optical modalities (i.e., diffuse optical tomography, ${ }^{5}$ fluorescence imaging, ${ }^{21}$ and spectroscopy), OCT is label-free and offers quantitative depth-resolved tumor information, making it well suited for cancer detection and diagnosis. Thus, the use of OCT for cancer detection and diagnosis has been extensively explored. The tremendous value of OCT for effective detection and diagnosis of cancer was first demonstrated through in vitro cell imaging, followed by a series of in vitrolex vivo and in vivo studies on a broad spectrum of malignancies including breast cancer, brain cancer, lung cancer, gastrointestinal (GI) cancer, bladder cancer, skin cancer, oral cancer, laryngeal cancer, and cervical cancer, to name a few. Here, we briefly review each of these.

\subsection{In Vitro Cell Imaging}

The depth of our understanding of oncology is dependent in large part on our ability to visualize and track the associated dynamic cellular changes. OCT provides the appropriate spatiotemporal scales for the nondestructive investigation into the complex oncological cellular dynamics in vitro. Using OCT, a variety of cell processes, such as chemotaxis migration, proliferation, deadhesion, and cell-material interactions, were investigated in thick tissue models. ${ }^{22,23}$ In addition, OCT can be used to monitor time-dependent changes associated with premalignant cells in vitro. ${ }^{24}$ For instance, OCT has been used to monitor the ratio of fibroblasts to mammary epithelial cells, which is instrumental in determining organoid phenotypes. ${ }^{24}$ Further study adapted the principles of dynamic light scattering to OCT to monitor the dynamics of intracellular motions of different cells (i.e., the viable and apoptotic cells). ${ }^{25}$ It was found that the rate of intracellular motion in apoptotic cells was higher than in more viable cells due to the remodeling of the cytoskeleton required for membrane blebbing and cell fragmentation. ${ }^{25}$ Among others, the feasibility of using ultrahigh-resolution fullfield optical coherence microscopy (FF-OCM) for identifying cancer cells has been investigated by measuring the refractive index distribution across a single live cell. ${ }^{26}$ Experimental results showed that cancer cells have a higher refractive index than normal ones, demonstrating that FF-OCM has significant potential for cancer diagnosis and dynamic cell analysis as an in situ label-free biophysical assay. ${ }^{26}$

\subsection{Breast Cancer}

Breast cancer starts internally from different parts of the breast, and the internal location of most breast lesions limits OCT applications to open surgeries or requires the use of needle-based imaging probes. ${ }^{27-36} \mathrm{In}$ addition, during breast-conserving surgeries, axillary lymph nodes that filter lymph fluid draining from the primary tumor site are removed for disease staging. Although a high number of lymph nodes are often resected during sentinel and lymph node dissections, only a relatively small percentage of nodes are found to be metastatic, a fact that must be weighed against potential complications, such as lymphedema. ${ }^{37}$ Therefore, the reported OCT study for breast cancer is largely focused on the differentiation of normal versus cancerous breast tissues with different subtypes for "optical biopsy," accurate surgical margin detection, and precise evaluation of axillary lymph nodes for breast cancer staging purposes.

In 2004, the first ex vivo OCT images of a carcinogeninduced rat mammary tumor model were reported. ${ }^{27}$ Ex vivo OCT images of early- and late-stage tumors and cellular features along tumor margins were also reported, showing their strong correlations with corresponding histopathology. The OCT images revealed structures of individual cells and identified suspicious isolated cells adjacent to the solid tumor that showed evidence of increased scattering, compared with the surrounding low-scattering adipocytes. The potential of OCT to be an effective tool for the detection of lesions in the human breast was confirmed with further studies. ${ }^{4,38}$ Comprehensive work has also been completed to establish the correspondence of OCT image findings with histopathologic findings to better understand, which features characteristic of breast lesions can be visualized with OCT. ${ }^{39}$ It was found that the microstructure of normal breast parenchyma, including glands, lobules, and lactiferous ducts, and stromal changes associated with infiltrating cancer, were visible, as well as fibrocystic changes from benign fibro-adenomas. Imaging of ductal carcinoma in situ, infiltrating cancer, and microcalcifications was correlated with corresponding histopathologic findings. This work and others highlight that OCT offers the potential for visualizing breast lesions at 
a resolution greater than that of currently available clinical imaging methods. Additionally, integrated OCT and OCM were reported for ex vivo multiscale evaluation of human breast tissues. ${ }^{40}$ Beyond providing distinctive patterns for adipose tissue, fibrous stroma, breast lobules and ducts, cysts and microcysts, as well as in situ and invasive carcinomas, OCT/OCM with its 3-D imaging capability provided complementary information to individual 2-D images, thereby allowing tracking of features from different levels to identify low-contrast structures that were difficult to appreciate from single images alone. ${ }^{40}$ To capture human breast OCT images in one shot, a benchmark pilot study was conducted in which FF-OCT was used to image human breast tissue. ${ }^{41}$ Features resulting from pathological modifications were characterized, a diagnosis decision tree was developed, and using FF-OCT images, two breast pathologists were able to distinguish normal/benign tissue from lesions with a sensitivity of $94 \%$ and $90 \%$, and specificity of $75 \%$ and $79 \%$, respectively, for the two pathologists. ${ }^{41}$ Traditionally, the penetration depth of OCT is somewhat limited ( $\sim 1$ to $2 \mathrm{~mm}$ ), potentially impacting its clinical utility. However, the development of various needle probes has enabled OCT imaging deep below the tissue surface. ${ }^{27-30,32-36}$ For example, a forward-viewing needle device with an integrated fiberoptic probe was developed to couple with an OCT imaging platform for the microscopic guidance of breast needle biopsy procedures. ${ }^{31}$ Since the probe is capable of assessing optical refractive index and scattering that are highly correlated with pathology, a diagnostic sensitivity of $89 \%$ and specificity of $78 \%$ were achieved to differentiate tumor from adipose breast tissues. ${ }^{31}$ Using a miniaturized side-viewing needle probe, the first published image of a human breast cancer tumor margin and of human axillary lymph nodes was also presented..$^{33} \mathrm{~A}$ clear distinction between an area of dense tumor and the honeycomb structure evident in the surrounding adipose tissue was identified in the OCT image of the tumor margin. In addition to the academic efforts to utilize OCT for breast cancer detection, scientists from industry have also been contributing to the same goal. ${ }^{32,42}$

Unlike the previously reported OCT studies that rely on the structural analysis of breast tissue, optical coherence elastography (OCE) probes tissue mechanical contrast in tissues ${ }^{35,43-47}$ and polarization-sensitive OCT (PS-OCT) is sensitive to form birefringence in human breast tissue. ${ }^{48,49}$ A dynamic spectraldomain OCE imaging technique with improved acquisition and processing speed has been reported. ${ }^{45}$ At different driving frequencies, this technique provides contrast among sample regions with different mechanical properties and thus is used to mechanically characterize tissue in a fast manner. ${ }^{45} \mathrm{Ex}$ vivo experiments showed that in heterogeneous rat tumor tissue, different tissue types were selectively highlighted under different driving frequencies according to their different resonances. Specially, under $45-\mathrm{Hz}$ excitation, the OCE image highlights predominantly the adipose tissue region, whereas under $313 \mathrm{~Hz}$, the OCE image highlights predominantly the denser, more stiff tumor tissue region. Furthermore, it has been found that OCE could identify regions, which were poorly differentiated in the OCT scattering image. ${ }^{45}$ Unlike external OCE techniques, which may suffer from an inability to maintain a sterile in vivo environment, an internal and dynamic OCE method, namely acoustomotive OCE (AM-OCE), was introduced and studied to show the feasibility of OCE techniques using an internal excitation method. This technique may also have the advantage for exciting and studying local biomechanical properties such as in the cancer microenvironment, instead of bulk excitation. ${ }^{44}$ OCE measurements deep within human tissues using needle OCE was also demonstrated ex vivo, extending the potential of OCE as a surgical guidance tool. ${ }^{35}$ An improved form of OCE was recently presented, named optical coherence microelastography (OCME), which is sensitive to the microscale variations in the mechanical properties of tissue. OCME has been shown to identify invasive tumor through distinctive patterns in the OCME images with enhanced contrast compared with standard OCT. ${ }^{50,51}$ As is consistent with standard OCT, OCME could identify adipose, smooth muscle, ducts, blood vessels, and tumor revealed by the microelastogram that was generated. In addition, ducts and blood vessels appeared as regions of high negative strain, indicating that they were more compressible than surrounding tissue. It was concluded that OCME reveals contrast in malignant tissue that is complementary and, in some aspects, superior to OCT.

Using a different variant of OCT, the capability of PS-OCT to differentiate ex vivo human breast tissue was investigated. ${ }^{49} \mathrm{PS}-$ OCT images of breast cancer (i.e., invasive ductal carcinoma) exhibit low birefringence and a uniform polarization state, reflecting the severe fragmentation of the collagen structures associated with the invasiveness of the human breast cancer. ${ }^{49}$ The reported work also demonstrates the feasibility of using PS-OCT to supplement structural OCT as a possible method for intraoperative margin detection. ${ }^{49}$ Similarly, deep volumetric imaging of human breast birefringence through the integration of side-viewing fiber-optic needle probes with PS-OCT was successfully realized. ${ }^{36}$ Oblique sections from the imaged tissue cylinders that match the plane of the histological sections were retrieved to directly compare the observed birefringence features to histology (Fig. 1), enabling the clear identification of zones of low birefringence and uniform structural signal as areas of malignant tissue, as well as the association of the higher birefringence signal with noncancerous, desmoplastic, or stromal regions. This clear match with histology confirms that birefringence can serve as an intrinsic biomarker to differentiate malignant from surrounding connective tissue, a milestone for the clinical implementation of the PS-OCT technique. ${ }^{36}$ Finally, photothermal OCT was developed with its functionality demonstrated using ex vivo human breast tissue. $^{52}$

For the evaluation of axillary lymph nodes, the first reported OCT images showed detailed morphological structure with corresponding histological features of lymph nodes from a carcinogen-induced rat mammary tumor model, as well as from ex vivo human lymph nodes containing late stage metastatic disease. ${ }^{28}$ In the same year, OCT was used for the intraoperative ex vivo imaging and assessment of axillary lymph nodes. ${ }^{53}$ Distinct scattering changes in the cortex of the lymph node were found, which can be used to differentiate normal from reactive and metastatic nodes, and these optical scattering changes were correlated with the inflammatory and immunological changes observed in the follicles and germinal centers. ${ }^{53}$ The capability of OCT to image nodal microarchitecture was also reported. ${ }^{54}$ Using three-dimensional OCT (3D-OCT), the first longitudinal study of 3-D transcapsule OCT imaging was performed on intact lymph nodes from inbred female rats, demonstrating microstructural changes during metastatic infiltration, and promoting OCT as a possible technique for intraoperative, real-time in situ 3-D optical biopsy of lymph nodes for the intraoperative staging of 


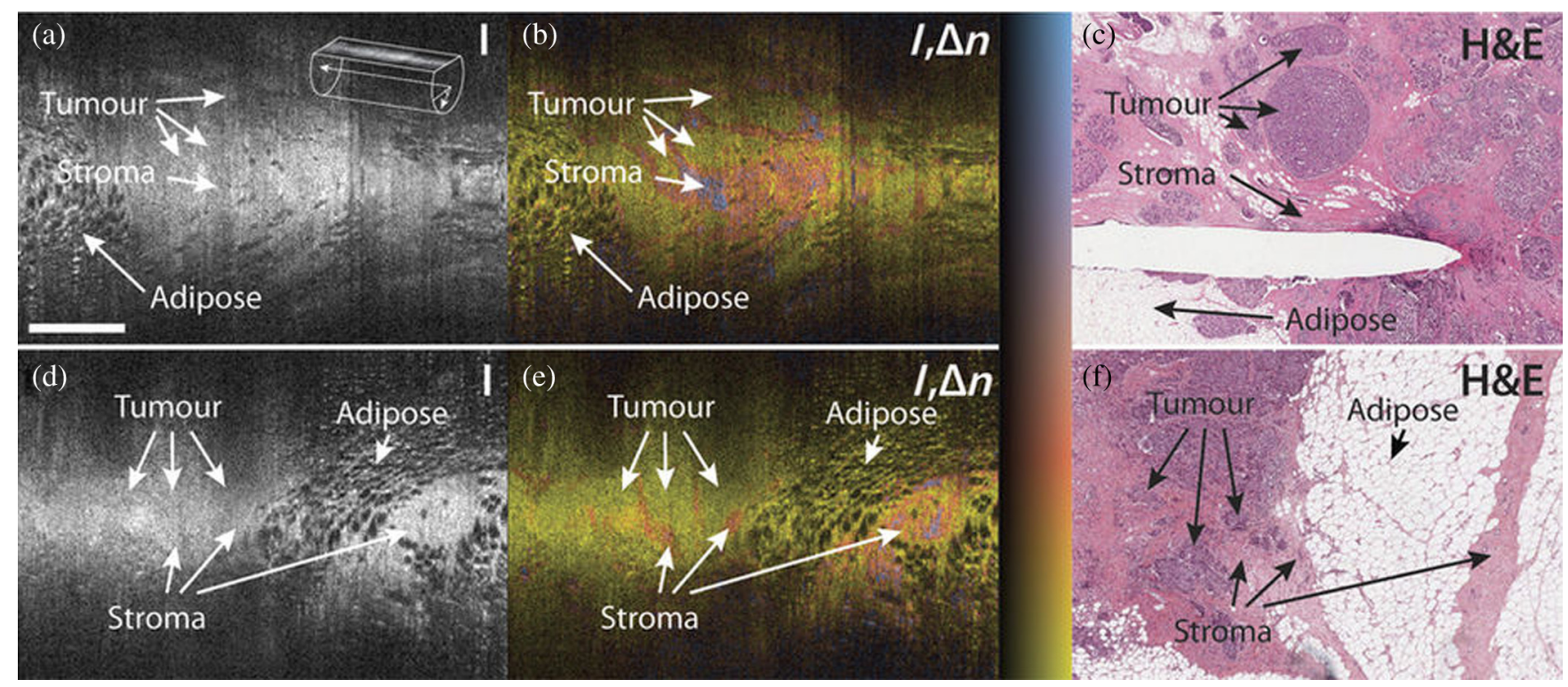

Fig. 1 Comparison of oblique cross sections with matching histology of ex vivo breast tumor samples measured using PS-OCT. (a, d) Structural intensity (l), (b, e) overlay of tissue birefringence and intensity and $(c, f)$ matching histological section stained with haematoxylin and eosin $(H \& E)$. (a-c) are from a widelocal excision (lumpectomy) of a $20 \mathrm{~mm}$, grade 1 invasive ductal carcinoma. (d-f) are from a mastectomy of a $20 \mathrm{~mm}$, grade 2 invasive ductal carcinoma. The needle track visible in (c) is due to a needle inserted into the tissue after imaging to guide the collection of histology, and hence does not appear in the OCT images. Scale bar is $1 \mathrm{~mm}$ and applies to all panels. Color range indicates birefringence of $0.18 \times 10^{-3}$ to $2.2 \times 10^{-3}$. Brightness range is 10 to $40 \mathrm{~dB}$. Figure reprinted with permission from Ref. 36

cancer. ${ }^{55}$ Recently, intraoperative 3-D OCT for assessing human lymph nodes for metastatic cancer was demonstrated. ${ }^{55}$ To further improve the image contrast between malignant and healthy nonneoplastic tissue in OCT images, parametric imaging of the local attenuation coefficient in human axillary lymph nodes was assessed using OCT. ${ }^{56,57}$

To advance OCT-based detection and diagnosis of human breast cancer and to reduce the nondiagnostic sampling rates, sophisticated algorithms have been developed by various research groups to accurately detect breast lesions ex vivo. For example, automated human breast tissue classifications with confidence-rating schemes were applied on the measured optical refractive index and scattering. ${ }^{31}$ OCT image segmentation for human breast cancer diagnostics was also demonstrated. ${ }^{58}$ In parallel, fractal analysis was proposed for classification of breast carcinoma using OCT $^{59}$ while an automated algorithm for differentiating adipose tissue from fibro-glandular human breast tissue based on parameters computed from the LCI signal (slope, standard deviation, and spatial frequency content) was demonstrated. ${ }^{30}$ Others have reported an automated breast tissue differentiation algorithm based on the parameters derived from OCT reflectivity profiles and the corresponding means and covariance matrices. ${ }^{60}$

During breast-conserving surgical procedures, achieving a clean surgical margin represents a technical challenge with important clinical implications. Currently, no label-free imaging method is available for surgeons to examine the in vivo resection bed or tumor cavity for microscopic residual cancer. The potential of OCT for the diagnosis of breast cancer has been demonstrated through previous in vitro/ex vivo experiments, but in 2015, the use of a handheld surgical OCT imaging probe was reported and demonstrated, for the first time, the in vivo use of OCT to assess tumor cavity margins, or the resection bed, for the microscopic presence of cancer. ${ }^{15} \mathrm{~A}$ total of 22 wide-local excision (WLE) patients were imaged, and three were found to have positive or "very close" margins (0 to $1 \mathrm{~mm}$ ) on histological analysis, whereas 10 were found to have cancer within 1 to $3 \mathrm{~mm}$ of the margin. Figure 2 shows the imaging results from a 72-year-old woman undergoing WLE for biopsy-proven invasive ductal carcinoma of the left breast. After excision of the primary WLE specimen, OCT videos and images were acquired from all six aspects of the resection bed by the surgeon using the handheld OCT probe. OCT imaging of the lateral aspect of the resection bed, of which one image is shown in Fig. 2(a), suggested a positive margin based on the microscopic architecture and scattering features present within the video data, which was confirmed as ductal carcinoma in situ on postoperative histological examination. Within the same surgery, an additional lateral margin specimen was removed and the surgeon again used the handheld probe to acquire OCT video images within the resection bed. OCT imaging of the lateral aspect of the resection bed after re-excision, of which one image is shown in Fig. 2(d), indicated a negative margin. In addition to the in vivo OCT assessment of the resection bed, ex vivo video images from the corresponding regions on the excised specimens [Figs. 2(b) and 2(c)] were also acquired during the WLE procedure. Strong correspondence was found between in vivo (before excision) and ex vivo (after excision) OCT images of the same tissue region [Figs. 2(a) and 2(c)] and postoperative histopathology. This work demonstrated the clinical use of real-time label-free video-based imaging of the in vivo resection bed following WLE to detect microstructural changes characteristic of residual cancer. The incorporation of a custom-designed handheld OCT surgical probe places the technology in the surgeon's hand for immediate assessment during the primary surgery. The ability to optically image, label- 


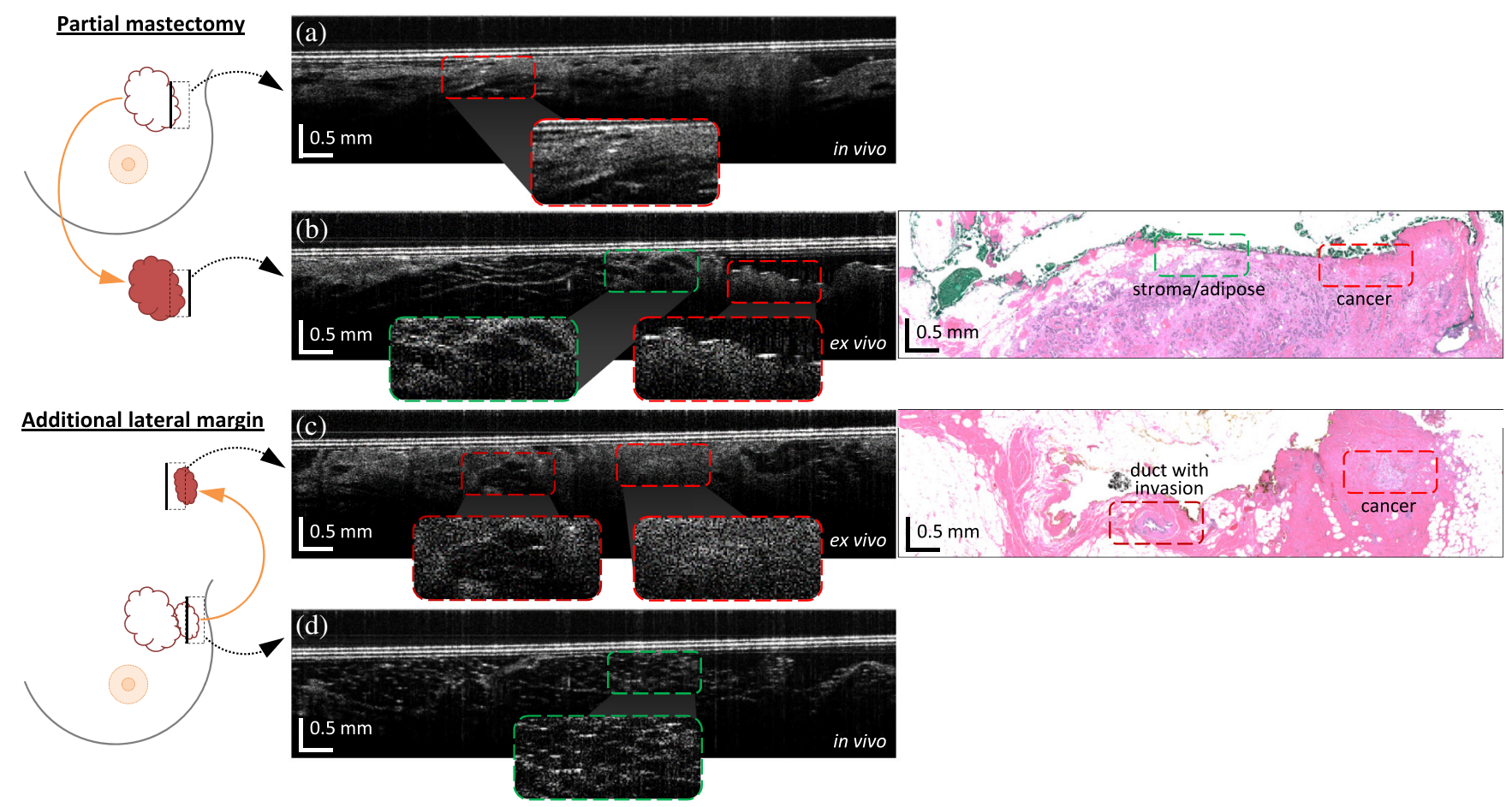

Fig. 2 Video OCT cross-sectional images of a positive tumor margin from the in vivo resection bed and ex vivo excised tissue. Images are from a 72-year-old female WLE patient with invasive ductal carcinoma of the left breast. Diagrams on the left indicate the imaged regions (dashed boxes) of the resection bed or the excised specimen (not to scale), and the solid black lines in the black dashed boxes indicate the top of the corresponding OCT image. The red and green dashed regions correspond to areas identified as cancer and normal areas, respectively. (a) OCT image of the positive in vivo lateral tumor margin. (b) OCT image of the positive ex vivo lateral specimen margin, with corresponding histology. (c) OCT image of the positive additional ex vivo lateral margin tissue [same tissue as imaged in vivo in (a)], with corresponding histology. (d) OCT image of the final negative in vivo lateral margin. Areas of interest are magnified and shown in the insets to compare normal stroma and adipose with cancerous regions. Note that histology images are only provided for the corresponding OCT images in (b) and (c), because the images in (a) and (d) were acquired in vivo and hence do not have corresponding histology images. Figure reprinted with permission from Ref. 15.

free, inside the tumor cavity and across the resection bed addresses the critical need for improved intraoperative detection of residual disease to ensure local control, and to potentially eliminate reintervention due to postoperative margin findings. With the development of various handheld and needle probes, coupled with portable OCT systems, OCT has the strong potential for routine use in the operating room, aiding surgeons to accurately assess the surgical margin and loco-regional lymph nodes, and thereby reduce the pain, anxiety, time, effort, and healthcare costs for patients diagnosed with breast cancer.

\subsection{Brain Cancer}

Several studies have focused on ex vivo brain tumor detection using OCT. ${ }^{16,61-70}$ With ultrahigh-resolution OCT (UHR-OCT), researchers have identified the morphological features, such as microcalcifications $(>20 \mu \mathrm{m})$, enlarged nuclei of tumor cells ( $\sim 8$ to $15 \mu \mathrm{m})$, small cysts, and blood vessels, which are characteristic of neuropathologies and normally absent in healthy brain tissue. ${ }^{64}$ The efficiency of UHR-OCT was further tested to image brain tissue morphology on a scale from single neurons to a whole animal brain using various animal models (i.e., bee, rabbit, and monkey) both ex vivo and in vitro. ${ }^{63}$ Acquiring images of a fixed rabbit brain slice, as one example, OCT clearly demonstrated the difference in optical scattering properties between gray and white brain matter, resulting in varying image depth penetration in the OCT images. The penetration depth was $\sim 1 \mathrm{~mm}$ in gray matter and diminished to less than $500 \mu \mathrm{m}$ in white matter. A series of work demonstrated that OCT allows discrimination of tumor adjacent to normal brain tissue, including diffuse and solid tumor tissue, and that OCT may be used to detect residual tumor within the cavity following resection of primary brain tumors through its integration with an endoscope. ${ }^{66,67}$

To demonstrate the potential for OCT imaging of brain within the surgical field, a portable handheld forward-viewing OCT surgical imaging probe was constructed. ${ }^{61}$ With the probe, cadaveric human cortex with metastatic melanoma was imaged in two- and three-dimensions, whereas 2-D images showed increased optical backscattering from regions of tumor, which was quantitatively used to determine the tumor margin. Three-dimensional reconstructions revealed regions of tumor penetrating normal cortex and could be resectioned computationally at arbitrary planes. Subsurface cerebral vascular structures could also be identified, to be avoided during surgeries. ${ }^{61}$ Likewise, with the use of a handheld forward-viewing OCT probe, the feasibility of OCT for differentiating cancer from noncancer was tested in human brain tissues ex vivo. ${ }^{16}$ The brain tissues were acquired from 32 patients with grades II and IV brain cancer and 5 patients with noncancer brain 
pathologies. It was found that pathologically confirmed brain cancer tissues (both high and low grade) had significantly lower optical attenuation values at both the core of the tumor as well as in the adjacent infiltrated zones, when compared with noncancer white matter. Using a diagnostic optical attenuation threshold of $5.5 \mathrm{~mm}^{-1}$, a specificity of $100 \%$ and a sensitivity of $92 \%$ was determined for high-grade tumors. For lowgrade tumors, the specificity was $80 \%$ and the sensitivity was $100 \%$ (9). Further, automatic positioning of the OCT probe through its integration with a robotic arm enabled OCT to precisely image the complete resection cavity of the brain tumor. ${ }^{68}$ Imaging ex vivo human brain tissues, the feasibility of using FF-OCT as a label-free nondestructive imaging technique in an intraoperative neurosurgical setting was demonstrated to assess tumorous glial and epileptic margins. ${ }^{70}$

The use of in vivo OCT detection strategies for brain tumors is still limited, with the majority of work being conducted on experimental animals. In 2002, the application of OCT for visualizing 1-D depth-resolved functional structures of cat brain was reported in vivo. ${ }^{71}$ To test the ability of OCT to detect cancer from noncancer intraoperatively and in vivo, five mice with two different high-grade human brain cancer xenografts were studied. ${ }^{16}$ Mice were implanted with either the U87 cell line or GBM272, a patient-derived cell line. OCT attenuation maps were acquired and displayed over the resection cavity during surgery, and it was found that OCT could resolve cancer versus noncancer regions on the scale of $0.004 \mathrm{~mm}^{3}$. OCT attenuation maps aided the user in identifying regions of cancer versus noncancer (white matter) before and after surgery, even for mice that displayed more infiltrative brain cancer characteristics with the patient-derived GBM272 cell line (Fig. 3). After imaging, mice brains were resected and the corresponding histological slides were reviewed by a neuropathologist for validation of the OCT results. These histological slides were sectioned in the same orientation as OCT cross-sectional images [that is, perpendicular to the tissue surface or perpendicular to (a)
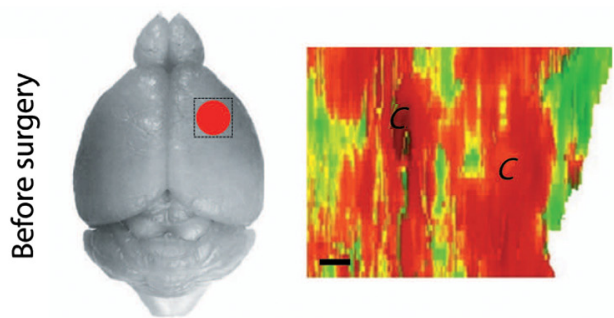

(b)

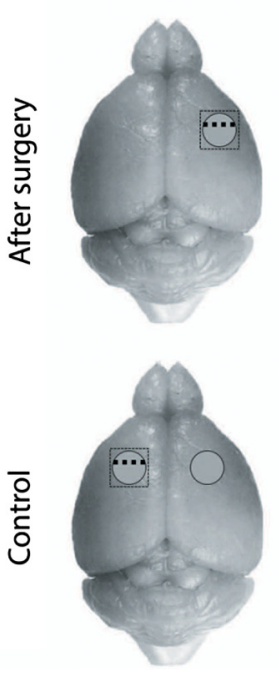

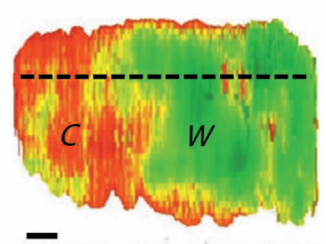

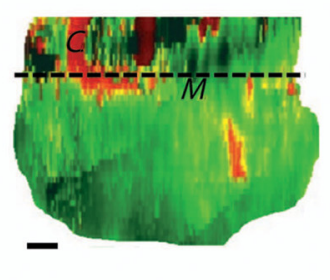

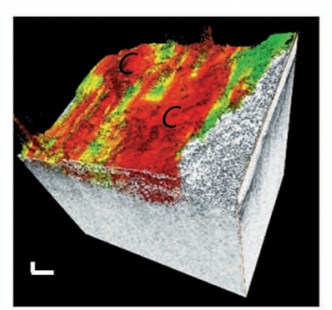

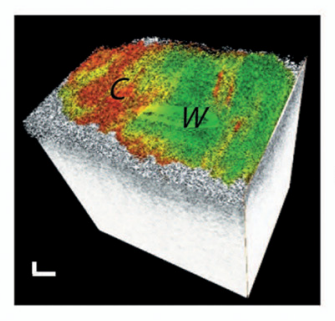

(c)

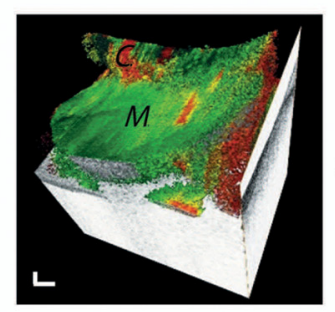

(e)

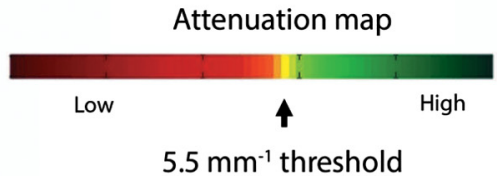

$5.5 \mathrm{~mm}^{-1}$ threshold
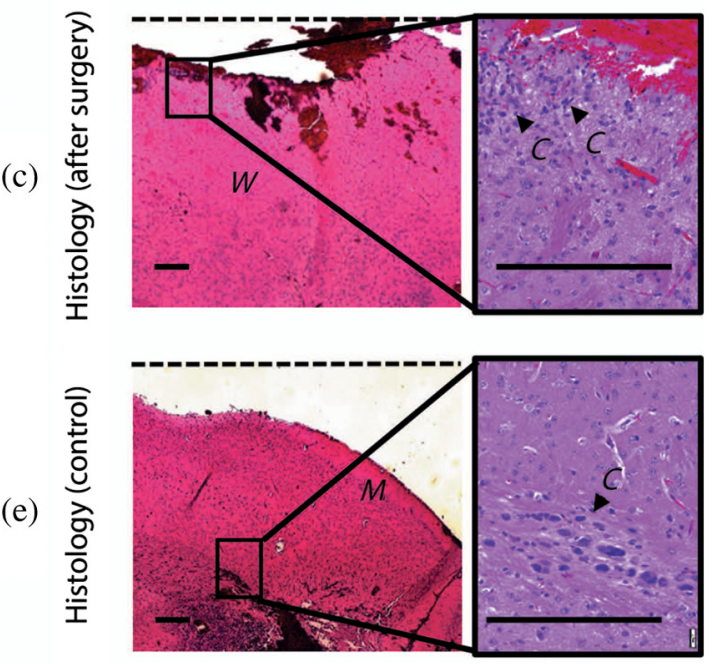

Fig. 3 In vivo brain cancer imaging in a mouse with patient-derived high grade brain cancer (GBM272). ( $\mathrm{a}$ and $\mathrm{b}$ ) Brain tissues were imaged in vivo in mice $(n=5)$ undergoing brain cancer resection. After imaging, the mice were sacrificed and their brains were processed for histology. The representative results of a mouse brain at the cancer site before surgery (a) and at the resection cavity after surgery (b) are shown. (c) Corresponding histology for the resection cavity after surgery. (d and e) From the same mouse, (d) control images were acquired from a seemingly healthy area on the contralateral side of the brain, (e) with the corresponding histology. The red circle indicates cancer, the gray circle indicates the resection cavity, and the square indicates the OCT field of view. Two-dimensional optical property maps were displayed using an attenuation threshold of $5.5 \mathrm{~mm}^{-1}$. Aliasing artifacts at the image boundaries, which were produced when dorsal structures from outside the OCT depth were folded back into the image, were cropped out of image. 3-D volumetric reconstructions were overlaid with optical property maps on the top surface. Optical attenuation properties were averaged for each subvolume of $0.326 \mathrm{~mm} \times 0.008 \mathrm{~mm} \times 1.8 \mathrm{~mm}$ within the tissue block, with a step size of $0.033 \mathrm{~mm}$ in the $x$-direction. Each histological image (c and e) represented a cross-sectional view of the tissue block: the image corresponds to a single perpendicular slice through the attenuation map, along the dotted lines in (b) and (d), respectively. Residual cancer cells were marked with black arrows and correspond to yellow/red regions on the attenuation maps (at the level of the dotted line). Abbreviations: $\mathrm{C}$, cancer; W, noncancer white matter; $\mathrm{M}$, noncancer meninges. Scale bars represent $0.2 \mathrm{~mm}$. Figure reprinted with permission from Ref. 16. 
the OCT attenuation map and along the dotted lines in Figs. 3(b) and $3(\mathrm{c})]$. In the postsurgery and control images, residual amounts of cancer (about 5\% to $10 \%$ of the imaged area) were visible in the histological images. ${ }^{16}$

The first in vivo OCT imaging of human brain was also reported. ${ }^{67}$ OCT images were obtained from different regions of the operating field at different stages of resection. The scarred cortical tissue on OCT showed high signal intensity with a rapid loss of the signal from deeper tissue structures. Correspondingly, a high attenuation coefficient of $5.3 \mathrm{~mm}^{-1}$ was found. When the resection proceeded to the solid and highly vascularized tumor, OCT analysis showed heterogeneous microstructure of the tumor parenchyma and a lower attenuation coefficient ranging from 0.9 to $2 \mathrm{~mm}^{-1}$. Histology showed a highly cellular tumor and neovascularization in this area. OCT analysis of tumor necrosis was characterized by low-intensity areas, focal cavernous-like structures, and low attenuation coefficients. ${ }^{67} \mathrm{In}$ addition, the combination of OCT with a surgical microscope was also reported for image-guided neurosurgery of brain tumors. ${ }^{72}$ The previous studies using OCT for brain cancer detection have shown its great promise to accurately detect brain tumor margins, and it is likely that more comprehensive in vivo human studies will be reported in the near future.

\subsection{Lung Cancer}

Ex vivo tissue analysis using OCT has also been performed on lung cancer samples, demonstrating the capability of OCT to image the microstructure of normal and abnormal bronchial tissue. ${ }^{73}$ Further, volumetric OCT of the normal airway allowed visualization of epithelium, lamina propria, cartilage, and alveolar attachments. Carcinomas exhibited architectural disarray, loss of normal airway and alveolar structure, and large optical attenuation. Squamous cell carcinomas showed nested architecture. Atypical glandular formation was appreciated in adenocarcinomas, and uniform trabecular gland formation was seen in salivary gland carcinomas. Mucinous adenocarcinomas showed alveolar wall thickening with intra-alveolar mucin. Interstitial fibrosis was visualized as signal-dense tissue, with an interstitial distribution in mild interstitial fibrotic disease and a diffuse subpleural pattern with cystic space formation in usual interstitial pneumonitis. ${ }^{74} \mathrm{~A}$ one-to-one OCT image of histopathology correlation study was also performed. ${ }^{75}$ Further study revealed an accuracy of $82.6 \%$ for lung carcinoma diagnosis using OCT. ${ }^{76}$ Notably, the advantages of PS-OCT for lung cancer imaging are highlighted for the detection of poorly differentiated carcinoma of the lung. ${ }^{77}$ Figure 4 shows PS-OCT of poorly differentiated carcinoma with surrounding fibrosis in ex vivo lung. Structural OCT [Fig. 4(a)] lacks the ability to clearly distinguish the areas of solid carcinoma from fibrosis. However, PS-OCT [Fig. 4(b)] visibly delineates the collagen-rich fibrosis from carcinoma, with high birefringence signal in the regions of fibrosis and a lack of birefringence in the adjacent solid carcinoma. The boundary between the solid carcinoma and fibrosis, which was clearly visualized with PS-OCT, was confirmed with matched histology [Fig. 4(c)]. These results suggest that PS-OCT could serve as a powerful imaging technology for assessing tissue acquisition sites within lung nodules by drastically enhancing differentiation between tumor and areas of fibrosis.
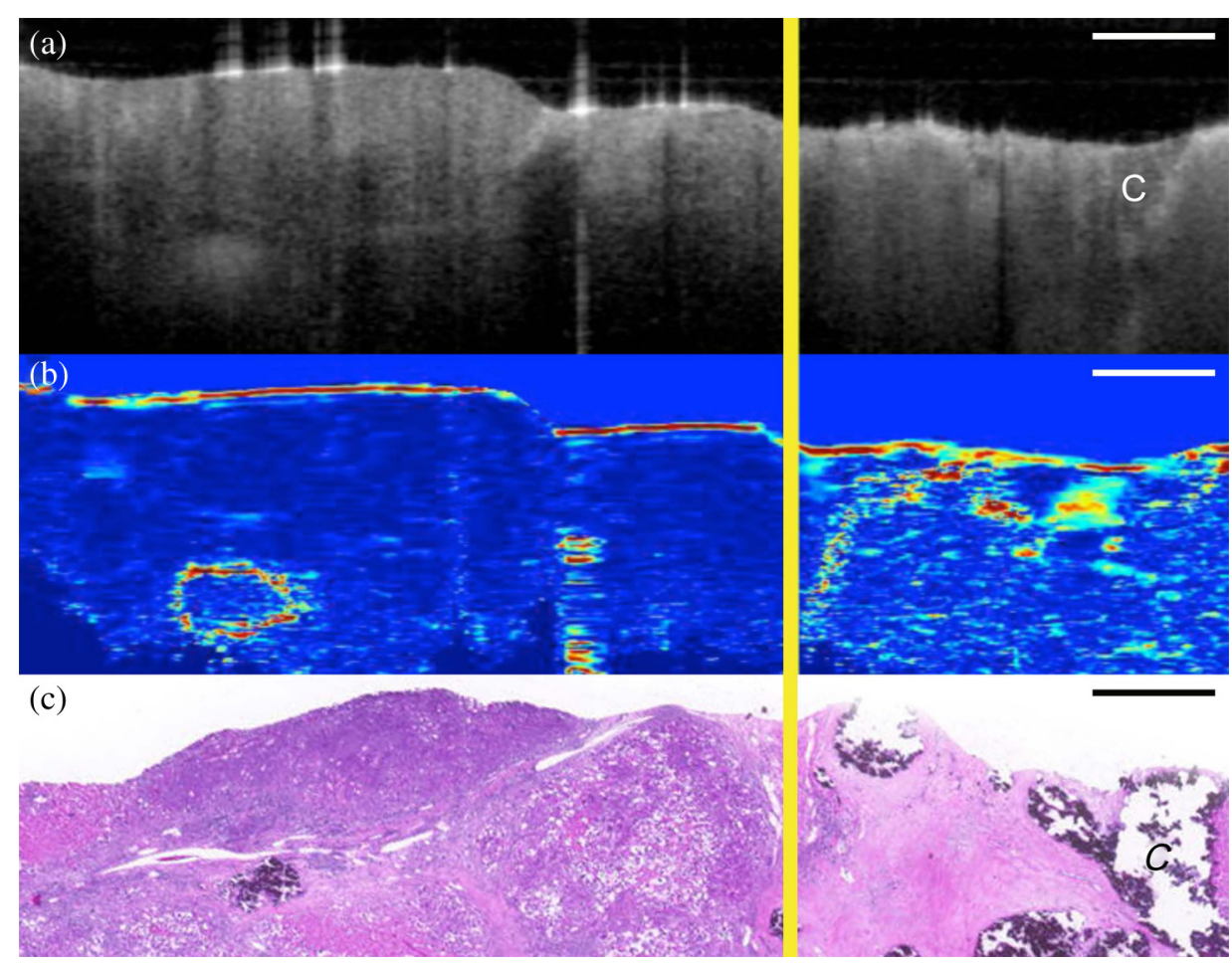

Fig. 4 PS-OCT of poorly differentiated carcinoma with surrounding fibrosis in ex vivo lung. (a) Structural OCT does not clearly show a distinction between solid carcinoma and adjacent fibrosis. Calcifications (C) within the fibrosis can be seen as signal-poor structures. (b) PS-OCT shows a clear delineation between solid carcinoma (left of line) and fibrosis (right of line), with no birefringence signal in the regions of carcinoma and high birefringence signal in the regions of fibrosis. (c) Matched histology confirming the demarcation between carcinoma and fibrosis. Scale bars represent $1 \mathrm{~mm}$. Figure reprinted with permission from Ref. 77. 
The characteristic features associated with normal and neoplastic bronchial tissues ${ }^{74}$ were also reported by the other peerreviewed works. ${ }^{78-80}$ Furthermore, OCT identified in situ morphological changes associated with inflammatory infiltrates. ${ }^{79}$ Inflamed tissue results in less well-defined OCT image features, where only the epithelium and lamina propria can be clearly delineated. However, OCT imaging captures the remodeling present within inflamed airways as visualized on histological analysis. ${ }^{79}$ The potential utility of FF-OCT in identifying and differentiating lung tumors from nonneoplastic lung tissue was also demonstrated. ${ }^{80}$ In addition to identifying lung tumor, various histological patterns of tumors could be identified in the FF-OCT images, especially for adenocarcinomas with various patterns.

The development of an OCT catheter probe that can pass through the working channel of medical bronchoscope has facilitated the in vivo detection of lung cancer using OCT. As early as 2003, a 2-mm diameter side-viewing OCT catheter probe was used to successfully measure the size and shape of the in vivo human upper airway. ${ }^{81}$ With the development of anatomical OCT, it was demonstrated that it was possible to record dynamic changes in airway size and shape with $\mathrm{OCT}^{82-84}$ and measure the elastic properties of the central airway in obstructive lung diseases. ${ }^{85}$ Further implementation of anatomical OCT on three different subjects such as one with subglottic tracheal stenosis, one with a malignant left main bronchus obstruction, and another with severe tracheomalacia, confirmed the potential of OCT to improve diagnostic and therapeutic bronchoscopy. ${ }^{13}$ With the use of a side-viewing needle probe to realize OCTbased in situ imaging of alveoli and the smaller airways, OCT has significant potential in the assessment of lung disease. $^{86}$ In 2005, catheter-based OCT was used for the in vivo diagnosis of a bronchial tumor. ${ }^{73}$ In 2008, microscopic OCT imaging of lung tissues was performed under the guidance of autofluorescence bronchoscopy. ${ }^{87}$ Quantitative measurement of the epithelial thickness using OCT showed that the epithelial thickness was significantly different between invasive lung cancer and carcinoma in situ $(p=0.004)$. Severe dysplasia and carcinoma in situ tended to be thicker than mild or moderate dysplasia, but the results did not reach statistical significance $(p=0.39)$. Taken together, mild, moderate, and severe dysplasia were significantly thicker than metaplasia $(p=0.002)$. Mild dysplasia tended to be thicker than metaplasia, but the results did not reach statistical significance $(p=0.069) .{ }^{87}$ With fiber-optic catheter-based OCT imaging, there will be increasing applications for the use of this technology in lung cancer and other respiratory tract diseases.

\subsection{Gastrointestinal Cancer}

With over 2,807,400 new cases of GI cancer (esophagus: 455,800; stomach: 951,600; and colon: 1,400,000) each year, ${ }^{88}$ it is not surprising that GI tissues have been heavily interrogated with OCT. The development of various catheter-based endoscopic probes for OCT imaging enabled GI cancer detection in vivo. However, initially, the feasibility of OCT for high-resolution imaging of GI malignancies was demonstrated with ex vivo imaging of normal and pathologic microstructures including Barrett's esophagus, a metaplastic condition that can progress to esophageal adenocarcinoma. ${ }^{89}$ Unlike the normal esophagus associated with stratified squamous epithelium, the uniformly layered structure is replaced by a metaplastic columnar epithelium in Barrett's esophagus, and this structural change can be identified in OCT imaging of Barrett's esophagus. ${ }^{89}$ The columnar epithelial morphology and other mucosal structures in normal colon were also distinctly visible using OCT. In contrast, disorganization of the normal mucosal layers and ulcerative lesions was identified in tissues from ulcerative colitis and colonic adenocarcinoma. ${ }^{89}$ In 1997, a group studied OCT imaging of the GI tract in vitro with human GI tissues harvested from surgical resection and autopsy specimens. ${ }^{90,91}$ The results showed that OCT images demonstrated clear delineation of the mucosa and submucosa in most specimens. Furthermore, microscopic structures such as crypts, blood vessels, or esophageal glands in the submucosa and lymphatic nodules were observed..$^{90,91}$

The feasibility of OCT imaging in the GI tract was also demonstrated for the diagnosis of specialized intestinal metaplasia (SIM) ${ }^{92}$ It was found that OCT images of SIM were characterized by: (I) an absence of the layered structure of normal squamous epithelium and the vertical "pit and crypt" morphology of normal gastric mucosa, (II) disorganized architecture with inhomogeneous tissue contrast and an irregular mucosal surface, and (III) presence of submucosal glands, which were seen as either pockets of low reflectance below the epithelial surface or invaginations through the epithelium. ${ }^{92}$

In 2005, ultrahigh-resolution OCT, characterized by an axial resolution of $<5 \mu \mathrm{m}$, and 3-D ex vivo OCT imaging of the large and small intestines, was reported with good correlations to histology. ${ }^{93}$ With ultrahigh-resolution OCT, it was possible to distinguish the epithelial layer of the mucosa and visualize individual villi, glands, and crypts. Architectural distortion of glands from inflammatory and neoplastic processes was also observed. Additionally, 3-D rendering enabled visualization of the surface pit pattern and mucosal folds, as well as subsurface crypt microstructure. ${ }^{93}$ In 2010, combined OCT/OCM was used to improve imaging depth and contrast, enabling cellular imaging of human tissues, including those in the upper and lower GI tracts. ${ }^{94}$ Coregistered OCM and OCT imaging was performed on fresh surgical specimens and endoscopic biopsy specimens, and images were correlated with histology. ${ }^{94}$ OCM enabled cellular imaging of specimens from the upper and lower gastrointestinal tracts over a smaller field of view, compared with OCT. Squamous cells and their nuclei, goblet cells in Barrett's esophagus, gastric pits and colonic crypts, and fine structures in adenocarcinomas were all visualized. OCT provided complementary information through the assessment of tissue architectural features over a larger field of view. Fundamental work in this area offered a baseline for interpreting future ultrahighresolution endoscopic OCT imaging of the GI tract. ${ }^{93,94}$

Although OCT provides detailed morphology of tissues, the complementary molecular information is not available. Therefore, OCT combined with different molecular imaging modalities have been investigated. For example, the feasibility of a combined OCT and laser-induced fluorescence (LIF) system for ex vivo imaging of mouse GI tissues has been demonstrated. $^{95}$ OCT successfully imaged samples of colon, esophagus, and small intestine, providing structural information about the tissues that correlated well with corresponding histological sections. For instance, unlike normal small intestine characterized by mild to moderate signal attenuation, the muscularis propria-serosa boundary was highly scattering, and clearly defined layered structures were observed in the OCT images. The small intestine affected by dysplasia or adenoma showed strong nonhomogeneous signal attenuation, and the 
involved muscularis propria was displaced out of the OCT field of view without clear tissue layers being identified. ${ }^{95}$ Additionally, however, LIF enabled characterization of the biochemistry of endogenous fluorophores in mouse colon, esophagus, and small intestine, providing information on collagen, $\mathrm{NADH}$, and hemoglobin. ${ }^{95}$ A pilot study on "ApcMin" mice was performed to preliminarily test fluorescence-guided OCT imaging for colon cancer screening. ${ }^{96}$ The OCT images acquired under the guidance of fluorescence imaging showed the normal appearance of the mucosa, submucosa, and muscularis propria layers with clear and distinct tissue boundaries. ${ }^{96}$ In contrast, the OCT images of the ApcMin mice that developed colonic adenomas showed changes in the morphology of the mucosal layer. These changes consisted of mucosal thickening over time (larger in older mice), or even growth of large polyps, and in some cases, the disappearance of the mucosa/submucosa boundary. The corresponding OCT images of ApcMin mice that developed colorectal adenocarcinoma showed the loss of tissue texture (absence of normal layers) and the presence of pockets of uneven reflective or dark areas, which were caused by high signal absorption in the necrotic tissue or by the presence of pools of mucus. ${ }^{96}$

With miniaturized OCT catheter designs, OCT imaging has also been performed endoscopically to visualize gastrointestinal tissue in vivo. ${ }^{9}$ First demonstrated in the in vivo rabbit GI tract, OCT has since paved a way to realize an "optical biopsy." $97-99$ In 2000, high-resolution imaging of the in vivo human esophagus and stomach was reported using OCT. ${ }^{100}$ With OCT, it was possible to discern in vivo mucosal structures including colonic crypts, gastric pits, and duodenal villi. It was also possible to discern the slight variations in the thickness of the esophageal mucosa. In addition, OCT images of the gut (i.e., esophagus, colon, and duodenum) wall were acquired in vivo, yielding the well-organized layered structures including the mucosa and the muscularis mucosae. ${ }^{100}$ Another study identified intramucosal carcinoma and high-grade dysplasia in Barrett's esophagus in vivo. ${ }^{101}$ Consistent with OCT imaging of SIM ex vivo, ${ }^{92}$ in vivo SIM was characterized by a lower surface epithelial scattering intensity compared with the subsurface scattering intensity, representing good surface maturation. Glands in SIM were regularly distributed and linear. For intramucosal carcinoma and high-grade dysplasia, glands were irregularly shaped, and the surface intensity was greater than the subsurface intensity, representing poor surface maturation. Notably, unlike previous OCT catheters that relied on endoscopic guidance to place the OCT catheter near or against the luminal wall, ${ }^{100,101}$ balloon-based OCT catheters can inflate and dilate the lumen of the tissue and center the OCT imaging optics, ${ }^{102-104}$ significantly advancing the use of catheter-based OCT imaging of the gastrointestinal tract. In vivo ultrahigh-resolution endoscopic OCT technique was also developed and demonstrated for colonic imaging in a mouse model. ${ }^{105}$ In addition, a comparative study between OCT and high-frequency catheter probe endoscopic ultrasound (CPEUS) was conducted. ${ }^{106}$ With OCT, microscopic structures such as esophageal glands, intestinal villi, colonic crypts, and blood vessels were easily identified using OCT. Mucosa and muscularis mucosa were clearly seen at all sites of the GI tract, except for the stomach, and OCT identified the submucosa in all of the sites of the GI tract. With CPEUS, mucosa and submucosa were not clearly discernible throughout the images, but it was possible to clearly identify the muscularis propria (and split into two layers) along with periesophageal adventitial tissue. Therefore, high-resolution OCT, despite a seemingly limited penetration depth, may be complementary to CPEUS with its higher penetration depth for clinical imaging of GI neoplasms. ${ }^{106}$

Although in vivo OCT imaging of the GI tract has been greatly facilitated by endoscopy, there exist certain inefficiencies limiting its impact. For most endoscopic procedures, subjects are sedated, requiring specialized settings, equipment, and medical staff to monitor for adverse reactions. To address this limitation, a swallowable tethered capsule OCT endomicroscopy (TCE) device has been developed. To summarize the results of this study, during the transit of the capsule, multiple OCT cross sections of the luminal organ are acquired at $30-\mu \mathrm{m}$ (lateral) $\times 7-\mu \mathrm{m}$ (axial) resolution, enabling the visualization of structures, such as normal squamous mucosa, Barrett's mucosa, and the gastric mucosa. ${ }^{107}$ Sequential cross sections were compiled to reconstruct a 3-D microscopic representation of the entire luminal organ [Figs. $5(\mathrm{~d})-5(\mathrm{~g})]^{107}$ Further 3-D fly-through movies show that the surface of the squamous mucosa is relatively smooth and has a higher OCT signal starting at the proximal end of the esophagus. Approximately at the midpoint of the OCT scan, a region of Barrett's mucosa was evident, which has a rough, irregular mucosal surface and a lower OCT signal. At the end of the fly-through movie, the gastric mucosa was imaged, where the mucosal surface was smoother than that of the Barrett's segment and rugal folds can be seen. The same group also developed a fully automated algorithm for the segmentation of in vivo OCT-TCE data sets and characterization of the esophageal wall. ${ }^{108}$

Functional OCT has also been implemented in vivo imaging in the GI tract. For instance, endoscopic Doppler OCT (EDOCT) has been reported for in vivo endoscopic imaging of blood flow in the rat and human GI tracts. ${ }^{109,110}$ Typically, normal esophagus was featured by small vessels in the lamina propria and medium vessels in the submucosa. Barrett's esophagus and esophageal adenocarcinoma were characterized by diffuse, small vessels in the superficial mucosa. Rarely, diffuse, small vessels were found in superficial mucosal layers of the normal stomach. Rather, diffuse, small vessels were more frequently found in the superficial mucosa of gastric lymphoma. ${ }^{109,110}$ These studies demonstrated that EDOCT can be useful for diagnostic imaging and treatment monitoring in the GI tract. ${ }^{109,110}$ The introduction of OCT into the field of GI oncology has been helping GI clinicians to better manage GI disease. It is foreseeable that the implementation of various functional OCT modalities in a fiber-optic probe or tethered capsule OCT endomicroscopy will significantly improve the detection accuracy of various GI neoplasms. In addition, multicenter large-scale studies are expected to further evaluate the diagnostic yield of OCT techniques for GI disease diagnosis.

\subsection{Bladder Cancer}

To evaluate the level of bladder tumor involvement, white-light cystoscopy in combination with transurethral resection remains the mainstay. However, $33 \%$ to $67 \%$ of urothelial carcinoma cases following these mainstay methods were under staged. ${ }^{111}$ Clinical evaluation of bladder cancer will benefit from real-time endoscopic diagnosis with the aid of OCT. In 1997, the potential of OCT to achieve "optical biopsy" of human urologic tissues was investigated. ${ }^{112}$ Different anatomical layers in the normal bladder were identified in the OCT image, including the mucosa, submucosa, and muscularis propria. In 1999, in vitro 


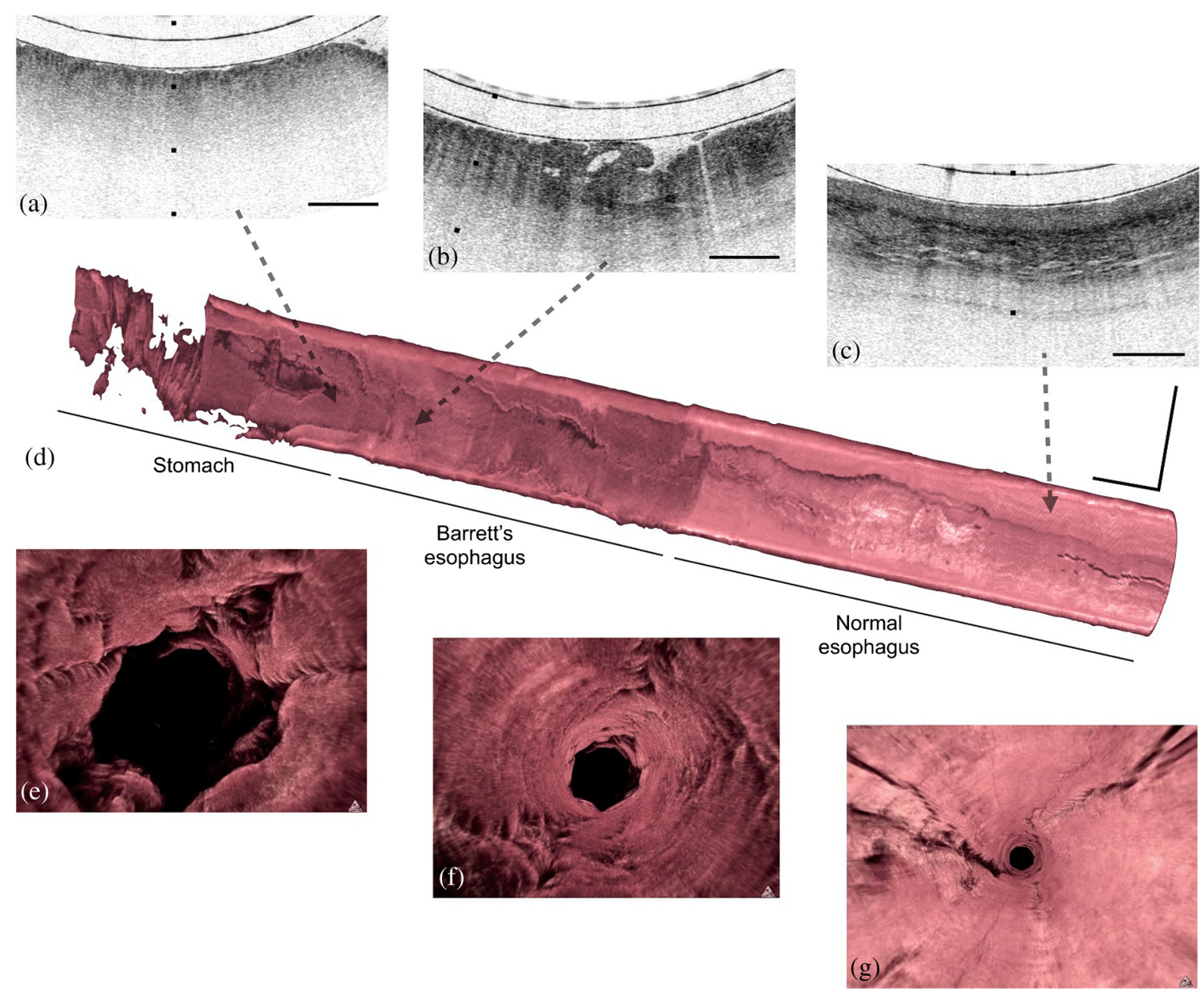

Fig. 5 Tethered capsule endomicroscopy data from a patient with a diagnosis of Barrett's esophagus and high-grade dysplasia, with intramucosal carcinoma. (a-c) Portion of a cross-sectional tethered capsule microscopy image of (a) the stomach, (b) Barrett's esophagus mucosa with architectural atypia suggestive of high-grade dysplasia, and (c) squamous mucosa at the distal, mid, and proximal ends of the esophagus, respectively. (d) A three-dimensional representation of the tethered capsule endomicroscopy data showing a 4-cm segment of Barrett's esophagus with multiple raised plaques and nodules, one of which corresponds to the features in (b). (e-g) Three-dimensional fly-through views of: (e) the stomach, (f) Barrett's segment, and (g) squamous mucosa showing a clear difference between the superficial appearance of the rugal folds of the stomach, the crypt pattern of Barrett's esophagus, and the smooth surface of the squamous mucosa. Tick marks and scale bars represent: $(a-c) 1 \mathrm{~mm}$; scale bars: (d) $1 \mathrm{~cm}$. Figure reprinted with permission from Ref. 107.

specimens were used to demonstrate the possibility of using OCT to distinguish between normal human bladders and those with invasive transitional cell carcinoma (TCC). ${ }^{8}$ It was noted that, unlike OCT images of the normal bladder, images of invasive TCC did not contain distinct layers or boundaries, and the study concluded that malignant invasion disturbed the normal well-defined strata seen in OCT images. ${ }^{8}$ In 2001, the first systematically organized OCT study of porcine bladder cancer was reported. ${ }^{113}$ OCT images of porcine bladder (a close homologue of human bladder) were first acquired, and it was found that the micromorphology of the porcine bladder, such as the urothelium, submucosa, and muscles, was readily identified by OCT and well correlated with the histological evaluations. Moreover, to determine whether OCT could track the longitudinal course of bladder cancer development, a standard rat model of bladder cancer was followed both with OCT and histological studies. It was shown that OCT could detect edema, inflammatory infiltrates, and submucosal blood congestion as well as the abnormal growth of urothelium (e.g., papillary hyperplasia and carcinomas). ${ }^{113}$ A comparative study was conducted between OCT and 40-MHz high-frequency ultrasound (HFUS) for imaging rat bladder cancer ex vivo, and the study suggested that multimodality cystoscopy combining OCT and HFUS may have the potential to enhance the diagnosis and staging of bladder cancers. ${ }^{114}$ Also, a 3-D time-lapse ultrahighresolution OCT (TL-uOCT) was developed for enhancing the diagnosis of nonpapillary bladder cancer. ${ }^{115}$ By proper timelapse frame averaging of snapshots of uOCT signals, the cellular details of bladder epithelium embedded in speckle noise could be uncovered. ${ }^{115}$ In 2006, a clinical study recruiting 50 patients with different clinical conditions of the bladder was reported, demonstrating the potential of OCT for improving the diagnosis of flat lesions of the urothelium. ${ }^{116}$ In 2008 , with OCT images acquired from healthy and morphologically altered bladder 
tissue ex vivo, the basement membrane (BM) zone of the bladder, which is identified as a narrow, low-scattering band between urothelium and lamina propria, was visualized. It was found that the existence of the BM zone allowed for reliable exclusion of bladder tumor invasion. ${ }^{117}$

The vast majority of bladder cancers originate within $600 \mu \mathrm{m}$ of the tissue surface, making OCT a potentially powerful tool for recognizing bladder cancers in vivo that are not easily visible with current techniques. In 1998, endoscopic OCT was demonstrated in the bladder where it was possible to discern the different layers (mucosa, lamina propria, and muscularis propria) of the bladder, along with blood vessels and cysts located within these layers. ${ }^{118}$ This study found that the urothelium of healthy bladder tissue appeared in OCT images as an area of low intensity, whereas the lamina propria appeared as an area of high intensity, and the muscle appeared as an area of low intensity, thereby providing contrast between layers. A microelectromechanical (MEMS) systems-based spectral-domain cystoscopic OCT system was developed to improve the diagnosis of early bladder cancer in humans. ${ }^{119,120}$ OCT identification of normal bladder was based on high-resolution delineation of bladder morphology. As shown in Fig. 6(b), urothelium appears as a thin, uniform, and low-scattering superficial layer, lamina propria-composed of mostly collagenous fibers - as highly scattering and heterogeneous, and upper muscularis as largely bifurcated collagen bundles, correlating well with histology [Fig. 6(c)] and consistent with previous OCT studies. ${ }^{118}$ The urothelial thickness measured by OCT $(89 \pm 8.3 \mu \mathrm{m})$ closely matched that of histology $(82.1 \pm 9.7 \mu \mathrm{m})$, and the interpatient variation was found to be insignificant ( $p>0.82, n=25$ ) if the bladder was distended properly, thus providing an important landmark for OCT diagnosis. ${ }^{119}$ OCT identification of TCC was based on enhanced urothelial heterogeneity and/or urothelial thickening attributed to random excessive growth of urothelial neoplasm. Figure 6(e) shows the result of a typical papillary TCC (pT1LG). Compared with normal bladder in Fig. 6(b), the architectural boundaries among urothelium, lamina propria, and upper muscularis were disrupted in the bladder tumor. ${ }^{119}$

To provide an automated diagnosis of bladder cancer based on OCT images, different methods have been explored. One automated algorithm used texture analysis to detect bladder cancer from OCT images. ${ }^{121}$ When compared with the corresponding pathology, the algorithm was effective at differentiating cancerous from noncancerous bladder tissue with a
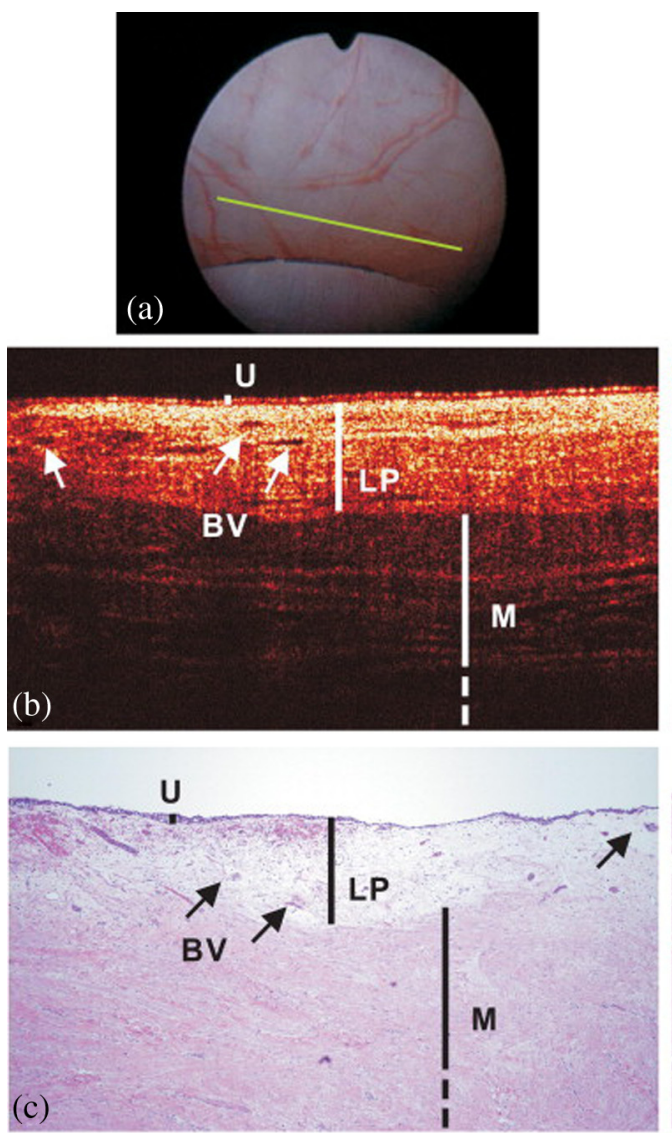
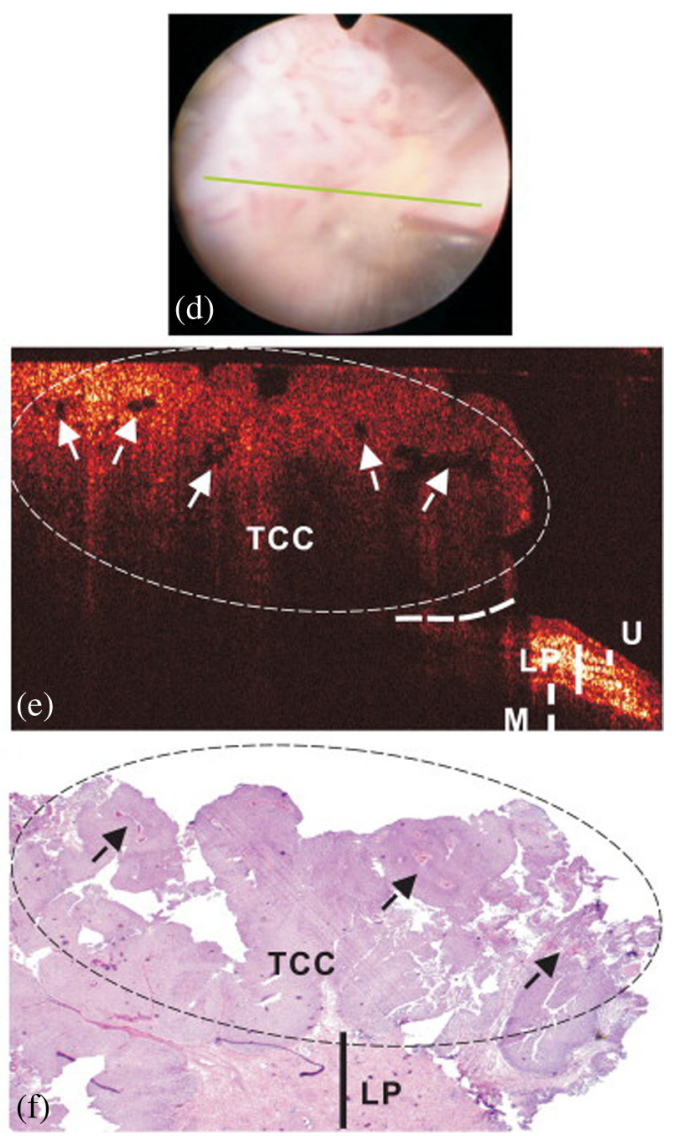

Fig. 6 In vivo surface, cross-sectional OCT, and H\&E-stained histological images of normal human bladder versus a papillary TCC. Image sizes: $\sim \phi 20 \mathrm{~mm}$ in (a, d) and $4.6 \mathrm{~mm}$ laterally by $2.1 \mathrm{~mm}$ axially in (b, e, c, and f). The morphological details of normal bladder (B), including urothelium (U), lamina propria (LP), and upper muscularis (M) were clearly delineated by OCT based on their backscattering differences, whereas those (LP, M) below the papillary TCC (e) are diminished. Solid arrows: subsurface blood vessels; dashed arrows: papillary features; dashed circle: TCC (low backscattering) identified by OCT based on increased urothelial heterogeneity; dashed line: boundary with adjacent normal bladder. Diagnoses of the normal bladder via OCT, cystoscopy, and histology were all negative, and voided cytology was positive. Diagnoses of a papillary lesion via OCT, cystoscopy, and histology were positive, while cytology was negative. Figure reprinted with permission from Ref. 119. 
sensitivity of $92 \%$ and a specificity of $62 \% .{ }^{121}$ Limited detection specificity was also reported in another study. ${ }^{122}$ First demonstrated in a rat bladder model, fluorescence-guided endoscopic OCT was shown to be helpful for enhancing early bladder cancer detection. ${ }^{123,124}$ Further work showed that combining fluorescence cystoscopy with targeted OCT imaging increases the detection specificity of bladder neoplasia with no added morbidity. ${ }^{125}$ Cross-polarization OCT (CP-OCT) was developed and introduced, along with a CP-OCT image metric called integral depolarization factor (IDF), to enable automatic diagnosis of bladder conditions (assessment the functional state of collagen fibers). IDF-based diagnostic accuracy for the identification of severe fibrosis of normal bladder mucosa was reported as $79 \%$, whereas the recurrence of carcinoma on the postoperative scar was $97 \%$, and the differentiation between neoplasia and acute inflammation was $75 \% .^{126,127}$

\subsection{Skin Cancer}

Skin cancer is common in humans, with basal cell carcinoma (BCC) being the most prevalent type. Surgery is the most common treatment for skin cancer; however, the identification of the tumor border is a challenge. Invasive punch biopsies of skin lesions are usually taken for histological diagnosis. Nevertheless, $\sim 20 \%$ of these biopsies misidentify aggressive subtypes of tumor due to sampling error. ${ }^{128}$ Therefore, noninvasive but high-resolution techniques such as OCT have been explored for skin cancer detection. ${ }^{129}$ Since skin is readily accessible for optical imaging, most of the reported OCT studies in skin cancer detection have been performed in vivo. The feasibility of OCT for BCC detection has been investigated, where a loss of normal skin architecture and derangement of the epidermis and upper dermis were observed in the OCT images. ${ }^{130}$ The investigators also found features that were frequently identified by OCT and correlated with histology including large plug-like signal-intense structures, honeycomb-like signal-free structures, and prominent signal-free cavities in the upper dermis. With regard to the aforementioned OCT features, no statistically significant $(p<0.05)$ difference was found between nodular, multifocal superficial, and infiltrative BCCs. ${ }^{130}$ The same team also used OCT to visualize and characterize melanocytic skin lesions in a systematic way. ${ }^{130}$ It was found that malignant melanoma often showed a marked architectural disarray $(p=0.036)$ and rarely displayed a clear dermoepidermal border $(p=0.0031)$ when compared with benign nevi. OCT of malignant melanoma infrequently demonstrated a dermoepidermal junction zone with finger-shaped elongated rete ridges as typically seen in benign nevi ( $p=0.011$ ). Compared with benign nevi, the papillary and superficial reticular dermis in malignant melanoma frequently displayed a more diffuse or patchy reflectivity with loss of the typical bright horizontal linear structures $(p=0.022)$. However, more or less large vertical, icicle-shaped structures were the most striking OCT features of malignant melanoma, which were not observed in benign nevi $(p<0.001) .{ }^{130}$

OCT has also been used to evaluate skin precancer (AK, actinic keratosis). ${ }^{131}$ Characteristic OCT image features reflected by the attenuation values were determined. Significant differences $(p<0.0001)$ were found between normal skin and AK using a gradient analysis on the attenuation values with $86 \%$ sensitivity and $83 \%$ specificity for the detection of $\mathrm{AK} .{ }^{131}$ One noted that aggressive forms of BCC are accompanied by extensive dermal invasion and destruction of collagen. The sensitivity of PS-OCT to collagen birefringence was first reported for BCC detection. ${ }^{132-134}$ Figure 7 shows examples of PS-OCT of BCC and healthy skin, with the corresponding histology. A healthy phase retardation image [Fig. 7(b)] shows an increase in the phase retardation values as a function of depth. The rate of this increase can vary by patient and location on the body. Despite this variation, most phase retardation images collected from healthy skin show roughly uniform phase retardation patterns in the lateral direction. BCCs in human skin manifest diverse patterns in phase retardation images. For example, a uniform pattern (similar to healthy skin) is observed in some subtypes of BCC, such as nodular BCC [Fig. 7(e)], whereas infiltrative BCC exhibits a different unique pattern [Fig. 7(h)]. ${ }^{134}$

\subsection{Oral Cancer}

Oral cancer is a common malignancy worldwide, with an annual incidence of over 300,000 diagnosed cases and an annual mortality of about 145,000 deaths. ${ }^{135}$ Patients with an advanced stage of oral cancer only have a $30 \%$ survival rate at 5 years, which can be improved to $80 \%$ to $90 \%$ if the oral cancer can be detected and diagnosed early for appropriate treatment. ${ }^{136}$ Since the oral cavity is easily accessible, most of the reported OCT applications in the oral cavity have been in vivo. However, an early study conducted in vitro examination of suspicious oral lesions (papules, ulcers, macules, plaques, and vesicles) using OCT. ${ }^{137}$ Four variables (changes in keratin, epithelial, and subepithelial layers, and the identification of the BM) were assessed from the oral OCT images and subsequently calculated whether or not there were architectural changes associated with oral lesions. It was found that basic histological layers (keratin, epithelium, and lamina propria) and microanatomical histological structures (including blood vessels, filliform papillae, and glandular ducts) were readily identified on most of the OCT images acquired from the oral lesions in vitro. Importantly, it was found thickening in the area of the "identified" BM as an indicative of invasion or extension by the tumor and can be one of the helpful image-based biomarkers in an OCT scan of oral tissues. A further ex vivo study confirmed the potential of the BM status changes in discriminating cancerous tumors from healthy tissue in the oral cavity. ${ }^{138}$ OCT images acquired ex vivo showed that the average epithelial layer thickness was variable and dependent on the pathological process. The mean thickness for the benign lesions was $338 \mu \mathrm{m}$, while for dysplasia (potentially malignant disorder) group, the mean thickness was $455 \mu \mathrm{m}$ for the epithelial layer. The mean thickness for carcinoma in situ on the OCT images was $570 \mu \mathrm{m}$, whereas in the presence of microinvasive carcinoma, the average thickness of the epithelium was $650 \mu \mathrm{m}$, which was higher than any of the other pathological processes. ${ }^{138}$ Further statistical analysis demonstrated that the thickness of the epithelium contributed to the ability of OCT to separate benign lesions from all other lesion types, ${ }^{138}$ yielding a sensitivity of $85 \%$ and a specificity of $78 \%$ in the assessment of potentially malignant and malignant disorders in the oral cavity.

In other works, SS-OCT was deployed to image oral lesion samples with different stages of cancer including normal oral mucosa, mild dysplasia, moderate dysplasia, early stage squamous cell carcinoma, and well-developed SCC. In dysplasia, it was determined that the boundary between the epithelium and lamina propria layers could still be identified and that the epithelium becomes significantly thicker compared with the normal control. However, in early stage squamous cell carcinoma and well-developed SCC stages, the epithelium-lamina propria 

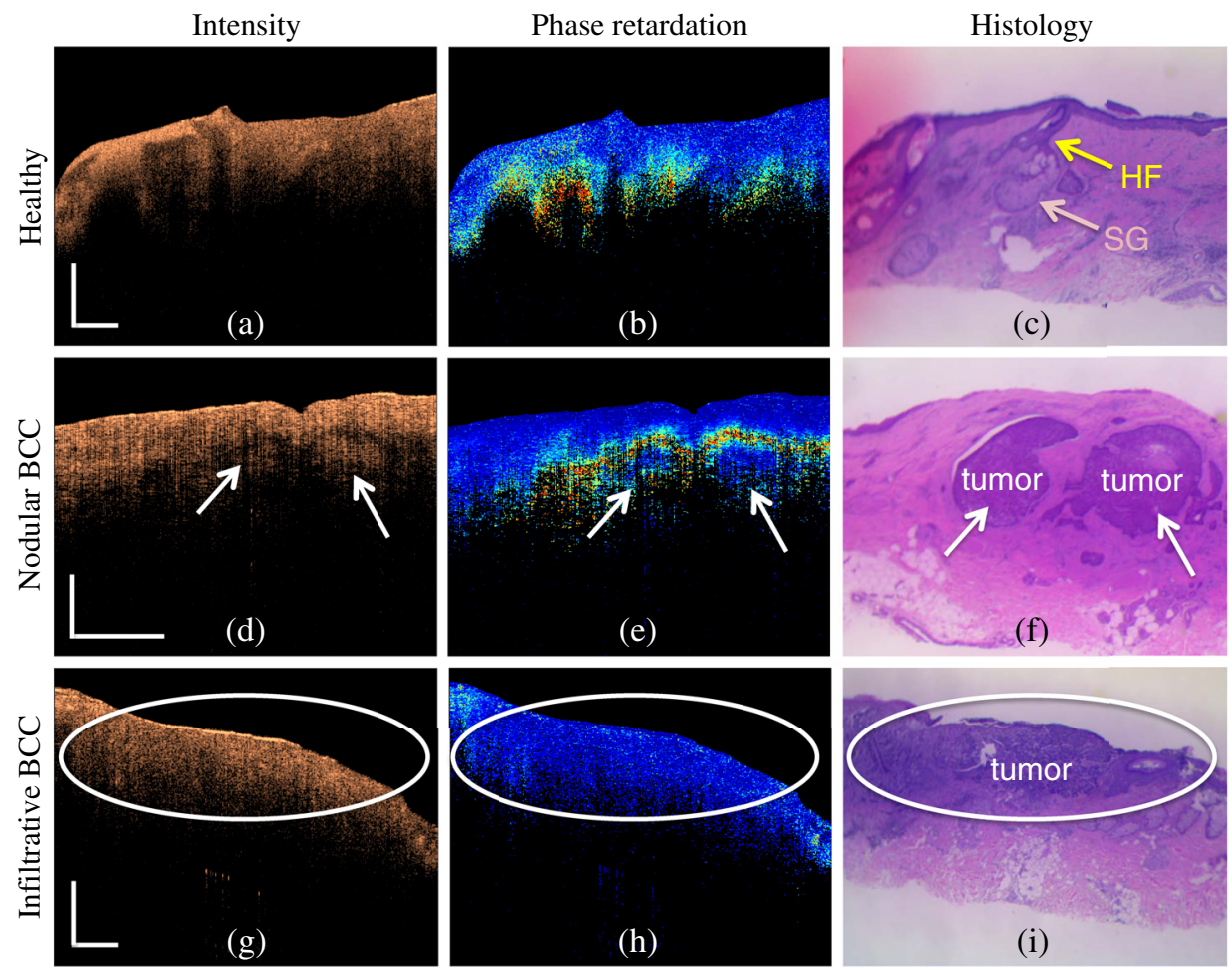

Fig. 7 (a) Representative intensity, (b) phase retardation, and (c) histology images of healthy and BCC human skin. (a), (b), and (c) represent the images of a healthy skin. Normal skin appendages such as a hair follicle (HF, yellow arrow) and sebaceous glands (SG, light pink arrow) are observed. (d), (e), and (f) images represent the case of a nodular BCC, and (g), (h), and (i) images represent a case of infiltrative BCC. The white arrows point to nodular tumor islands. Scale bars represent $500 \mu \mathrm{m} \times 500 \mu \mathrm{m}$ and are applicable to all images in a row. Figure reprinted with permission from Ref. 134.

boundary disappears. ${ }^{139}$ In addition, a preliminary study involving 50 patients with different oral lesions (i.e., dysplasia and malignancy) demonstrated the excellent capability of in vivo OCT for detecting and diagnosing oral premalignancy and malignancy in human subjects. ${ }^{140}$ A PS-OCT instrument capable of widefield in vivo imaging in the oral cavity was also developed, covering 2-D tissue imaging fields $\sim 2.5$-mm wide by up to 90 -mm length in a single image acquisition. ${ }^{141}$ In Fig. 8, PS-OCT images of an oral submucosal fibrosis lesion on the buccal mucosa of a 48-year-old male patient are shown. The en face image [Fig. 8(a)] is unremarkable except for some irregular vertical patterning near the right boundary of the lesion, which likely coincides with the anterior boundary of the lesion. This boundary is about $2 \mathrm{~mm}$ away from that denoted by the pink line and gives an indication of the precision of matching the clinical perception with the OCT imaging location. The azimuthal presentation along the solid (blue) horizontal line [Fig. 8(c)] shows an abnormal and very corrugated epithelial-submucosal boundary. The corresponding azimuthal retardation image [Fig. 8(f)] shows tight horizontal banding in the lesion region (i.e., between the pink lines), indicating high birefringence due to the underlying fibrotic tissue. Outside of the area of the lesion there is less birefringence. This is further demonstrated in the structural OCT and retardation Cartesian slices along the dotted (red) and dashed (green) lines [Figs. 8(d), 8(e), 8(g), and 8(h)].

\subsection{Laryngeal Cancer}

Although OCT images of a healthy larynx can be used to identify different layers, including the epithelial layer and lamina propria, ${ }^{142-149}$ the OCT images of laryngeal cancer generally show unrecognizable structures. ${ }^{142-149}$ With an OCT probe passing through the lumen of a surgical laryngoscope, it was found that the BM boundary between the epithelium and the lamina propria was lost with invasive carcinoma in the larynx. ${ }^{144}$ A transition zone was identified in the OCT images of the laryngeal tumor edge, which appeared as a gradual thickening of the epithelial layer and loss of delineation between the epithelial layer and the submucosal structures. ${ }^{144}$ In addition, an increased backscattering was observed from the area of tumor, indicated in the OCT images. ${ }^{144}$ Subsequently, the feasibility of using OCT to image the larynx in awake patients was demonstrated, ${ }^{147}$ as well as for monitoring laser surgery of laryngeal carcinoma. ${ }^{142}$

Although the stratification seen in OCT images was a criterion of healthy laryngeal tissue, the disappearance of such stratification was a sign of pathological alterations. In the case of carcinoma, OCT images revealed a lack of regular structure and the presence of a high backscattering area. ${ }^{142}$ The different imaging contrast shown in OCT images of healthy and cancerous laryngeal tissues makes it possible to accurately locate the tumor border during laser surgery of laryngeal carcinoma. Several studies have found that the thickness of the epithelium was the main criterion for the degree of dysplasia in the larynx. ${ }^{148}$ For instance, the thickness of squamous epithelium did not exceed $250 \mu \mathrm{m}$, whereas the average epithelial thickness can reach $530 \mu \mathrm{m}$ for severe dysplasia. Further, PS-OCT imaging of the benign and malignant larynx was investigated (204). Figure 9 shows the in vivo OCT images obtained from oral squamous cell carcinoma, showing clear boundaries between 


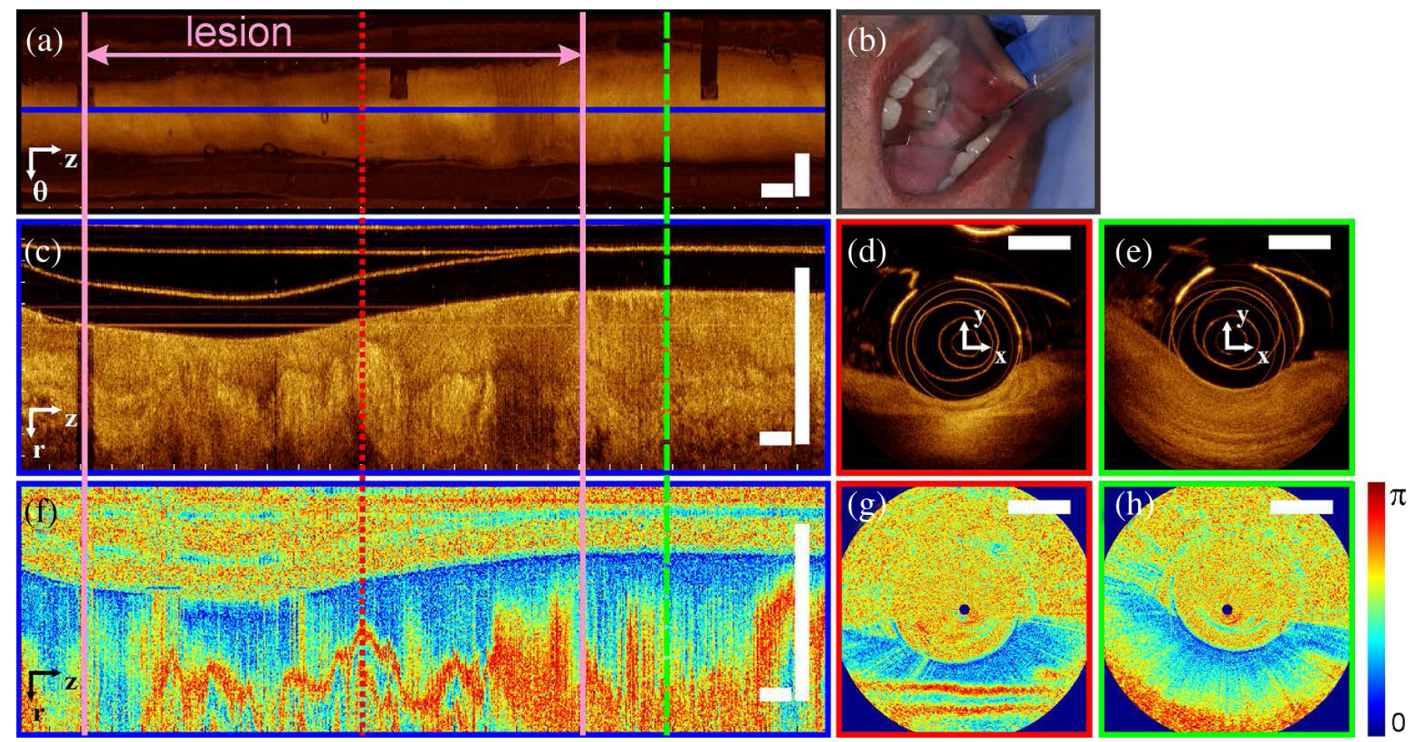

Fig. 8 OCT imaging of a submucosal fibrotic lesion on the buccal mucosa of a 48-year-old male patient. The pullback direction is from left to right corresponding to the posterior to anterior orientation. (b) Clinical photo of the lesion being imaged with the modified saliva ejector catheter holder. Structural OCT with (a) en face projection and (c) azimuthal slices. The clinically visible lesion is indicated between the solid (pink) vertical lines. Slices along the dotted (red) and dashed (green) lines are shown in Cartesian images in (d) and (e), respectively. (f, g, and h) Retardation images corresponding to the structural images (c, d, e), respectively. The images are averaged with a triangular kernel of width $5(w=5)$. The white bars in the images represent $1 \mathrm{~mm}$ in length. Figure used with permission. ${ }^{141}$

the normal vocal fold layered microstructure and the invasive cancer on both conventional OCT and PS-OCT. ${ }^{149}$

\subsection{Cervical Cancer}

To evaluate the performance of OCT for the diagnosis of cervical cancer, the first in vivo OCT imaging of human cervix was reported in 2003. ${ }^{150}$ It was found that normal and diseased microstructures such as capillaries, Nabothian glands, and in particular, the basal lamina, were identified at an unprecedented high resolution. ${ }^{150}$ Further work demonstrated that OCT images of invasive cervical cancer were characterized by an unstructured highly backscattering epithelium with a complete lack of layer architecture. Inflammatory changes were characterized by swelling and edema, blurring the boundary between the epithelium and the stromal layer. ${ }^{151}$ The reported sensitivity was high (>96\%), with a specificity of $\sim 60 \% .{ }^{151}$ A similar level of diagnostic specificity was reported for cervical intraepithelial neoplasia (CIN) $2,{ }^{152}$ showing substantial improvements compared with colposcopy and acetic acid whitening for cervical cancer screening in low-resource settings. ${ }^{152}$ A pilot study was also reported using OCT for the noninvasive examination of cervical tissue. ${ }^{153}$ OCT images were analyzed quantitatively for intensity of backscattered light from the epithelium and for rates of signal decay over the depth of the epithelium (slope). ${ }^{153}$ It was found that the average epithelial intensities were significantly greater in the abnormal tissue than in the normal tissue of premenopausal women $(p<0.0024)$ but were greater in the
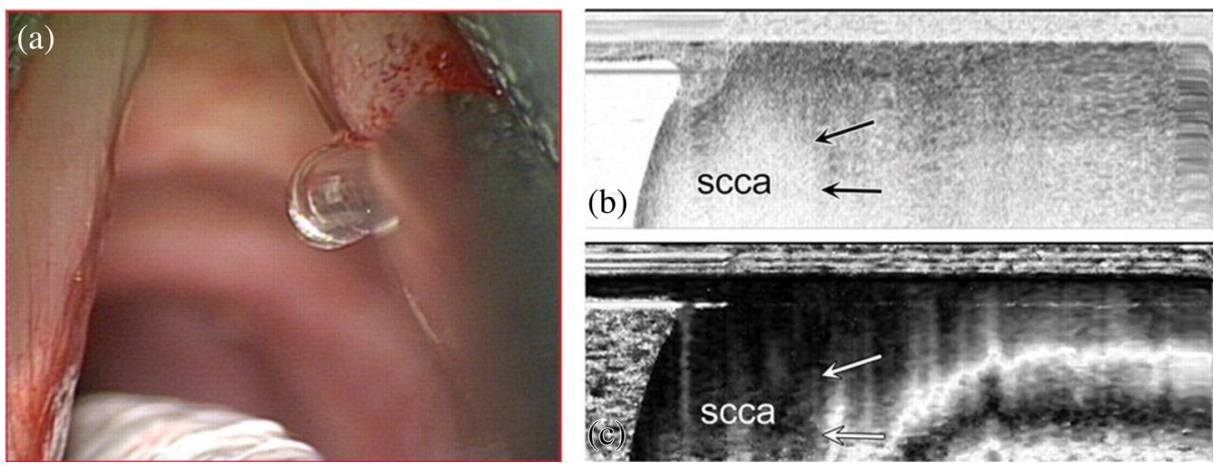

Fig. 9 (a) Intraoperative view of the glottic surface showing probe placement at the interface between the normal vocal fold and carcinoma on the right side. (b) Conventional OCT image shows a signal void in the area of cancer (scca) with sharp contrast to the gray scale image of normal tissue (arrows). (c) Clear distinction between the cancer, which is seen as a black area on the left side of the image, and normal vocal fold, which contains the light-dark-light banding pattern. Arrow depicts the clear boundary between cancer (scca) and normal. Figure reprinted with permission from Ref. 149. 
normal tissue of postmenopausal women $(p<0.062)$. No significant differences in signal decay rate were detected. ${ }^{153}$ A prospective study on the use of OCT in women with suspected CIN was later conducted, confirming the potential of OCT for improved differentiation of cancerous and precancerous lesions in the cervix. ${ }^{154}$ Recently, diagnostic efficacy of OCT for the precancerous cervix was investigated. ${ }^{155}$ As consistent with previous study, ${ }^{156}$ the healthy cervix exhibited a clear two-layered structure: the first epithelial layer being delineated from the stroma layer by a sharp boundary at the BM [Figs. 10(a) and $10(c)]$. The stroma is more highly scattering than the epithelium and therefore appears brighter in the OCT image. The thickness of the epithelium and the intensity contrast between the epithelium and the stroma vary little within a single OCT image of normal cervix. As CIN progresses, the two-layered structure becomes much less distinct and regular [Figs. 10(e), 10(g), 10(i), and $10(\mathrm{k})]$. The intensity contrast between the first and second layer decreases as well [Figs. 10(e), 10(g), 10(i), and 10(k)]. After epithelium segmentation \{i.e., [Figs. 10(b), 10(f), and $10(i)$ ] $\}$ and feature calculation, a sensitivity of $51 \%$ and a specificity of $92 \%$ were achieved to differentiate normal and CIN 1 versus inflammation and CIN 2/CIN 3. ${ }^{155}$

\subsection{Commercialization of OCT Systems for Oncology}

Overall, OCT offers oncologists an image-based instrument allowing real-time assessment of tissue pathology. More importantly, various commercial OCT imaging systems and OCT probes have been available in the market, a significant milestone for the adoption of OCT into the field of oncology. For instance, an OCT-based ex vivo specimen imaging system has been developed and commercialized for imaging breast cancer and skin cancer by Perimeter Medical and LL Tech. Michelson Diagnostics has commercialized OCT for imaging skin disease. In addition to the different commercial OCT imaging systems, handheld-, needle-, catheter-based-, and balloon-based-OCT probes have also been commercialized by different companies. Diagnostic Photonics is developing an intraoperative OCT system and handheld surgical imaging probe for in vivo imaging of the surgical cavity in breast cancer. Tomophase is developing catheter-based OCT probes for lung cancer imaging. NinePoint Medical is developing balloon-based OCT catheters for gastrointestinal cancer imaging, and MiniProbes is developing needle probes for brain cancer imaging. Progress made in both academia and industry has significantly advanced oncological imaging using OCT. Collectively, these advances in both the technology and the applications have demonstrated the possibility of using OCT for targeted biopsies and accurate evaluation of the resection margins and lymph nodes in the operation room, enabling the complete removal of tumor tissue and cells in one procedure, improving outcomes and survival rates, thereby effectively reducing medical costs and the health care burden to society.

\section{Monitoring of Cancer Therapy}

With enhanced cancer detection and diagnosis enabled by OCT, it was logical to consider how OCT could also be used to

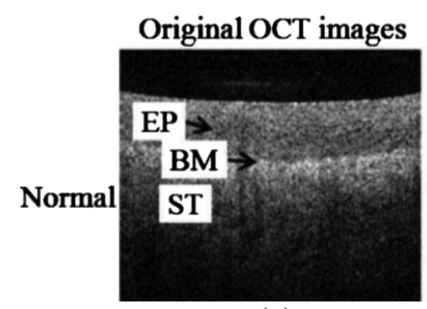

(a)

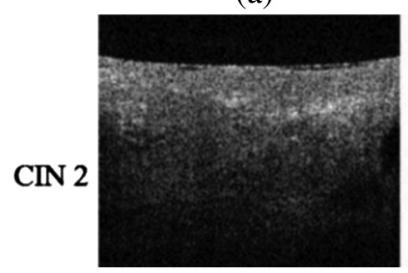

(e)

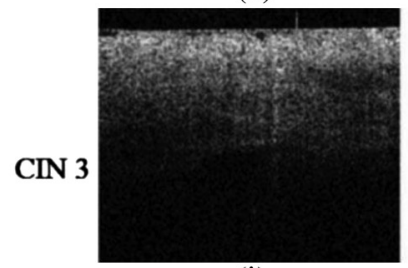

(i)

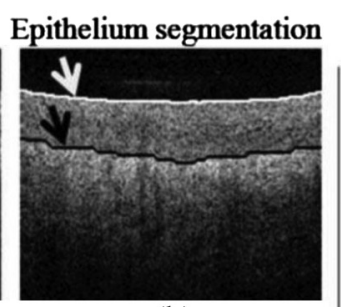

(b)

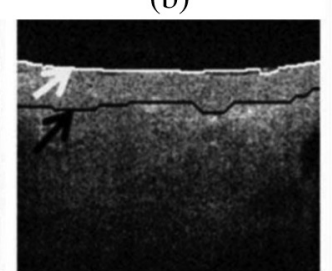

(f)

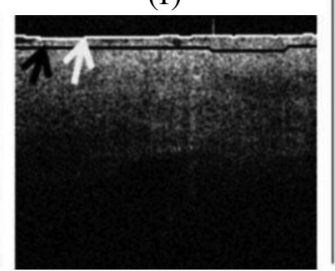

(j)

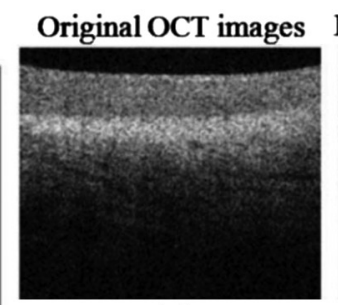

(c)

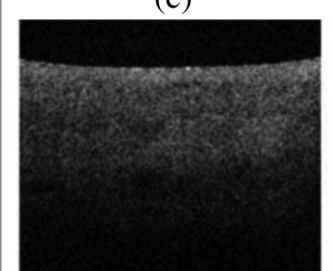

(g)

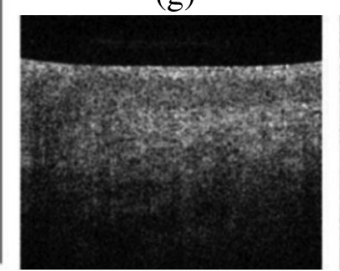

(k)

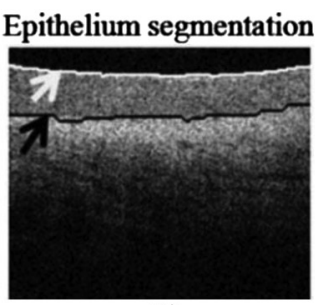

(d)

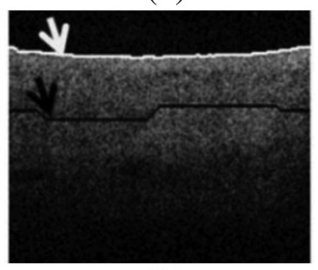

(h)

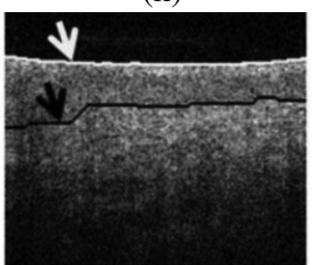

(1)

Fig. 10 Representative images of normal and high-grade CIN with epithelium segmentation results. White curves and white arrows indicate the probe-tissue interface segmented by the computer-aided diagnosis algorithm, and black curves and black arrows indicate the segmented boundary between the epithelium and stroma. (a)-(d) Typical normal cervical OCT images and segmentation results. All structures are well defined, including the epithelium (EP), the BM, and the stroma (ST). [(e)-(I)] OCT images of CIN 2 and CIN 3. The layered architecture becomes irregular or is not apparent. Images shown in (e) and (f), and (i) and (j) were correctly classified. Images shown in (g) and (h), and $(k)$ and $(\mathrm{l})$ were misclassified. Figure reprinted with permission from Ref. 155. 
monitor and determine effective cancer treatments for patients. Usually, chemotherapy, radiotherapy, ablative therapy (thermal, cryo/freezing), photodynamic therapy (PDT), and vasculartargeting therapies with or without surgery are the most common types of cancer treatments available. ${ }^{157,158}$ Considering that different therapeutic strategies exhibit different markers of an effective treatment response, ranging from alterations of micrometer-scale cellular features, to destruction of vascular networks, to morphological and biomechanical changes in cancerous lesions that can be tens of centimeters in size, monitoring these different treatment responses remains a critical aspect in optimizing the treatment of cancer, particularly since it is well recognized that tumors are also highly heterogeneous, with individual cancer cells responding to treatments in highly individualized ways.

With the ability to replicate tissue heterogeneity and treatment resistance, 3-D culture systems have been developed to closely replicate the dose-dependent treatment responses observed in patients, making them a valuable tool for evaluating and optimizing therapeutic regimens. ${ }^{159} \mathrm{OCT}$ is a good modality for 3-D culture studies as it enables continuous monitoring of the spatiotemporal dynamics in nonperturbative and nondestructive ways. ${ }^{23}$ OCT has been used to quantitatively monitor tumor burden in vitro throughout the course of PDT by mapping the volumes of individual 3-D tumors. ${ }^{160}$ With OCT, it was also possible to visualize the progression of apoptosis in vitro without labels via the elevated light scattering caused by chromatin condensation. ${ }^{160}$ In addition, quantitative tracking of cell death and viability in a 3-D tumor model of ovarian cancer in vitro with OCT has been reported. ${ }^{161}$ After treatment of ovarian cancer using PDT, cell death occurs via both apoptosis and necrosis, which can lead to the loss of cell-cell adhesion and cause structural disruption and fragmentation, in the case of 3-D nodules and tissues. Therefore, OCT measurements of the surface area and volume within a nodule can be used to identify necrosis. $^{161}$

During apoptosis, cells undergo a series of processes that package cellular contents into small dense vesicles, creating a local index of refraction mismatch. These vesicles also become strongly scattering bodies within 3-D tumor nodules that can be detected and quantified with OCT. ${ }^{161}$ The increased backscattering during apoptosis, and simultaneously the decreased light scattering associated with necrosis, has been confirmed by the reported work using OCT to investigate apoptosis- and necrosis-induced changes..$^{25,162,163}$ For instance, it was found that the optical attenuation coefficient in necrotic cells decreased from $2.2 \pm 0.3 \mathrm{~mm}^{-1}$ to $1.3 \pm 0.6 \mathrm{~mm}^{-1}$, whereas, in apoptotic cells, an increase to $6.4 \pm 1.7 \mathrm{~mm}^{-1}$ was observed. ${ }^{162}$ Similarly, another study reported a gradual decrease in scattering associated with necrotic cells, which probably stemmed from their continuous disassembly. ${ }^{163}$ To continuously visualize the structural changes within a sample over the course of hours, days, and even weeks, time-lapse OCT (TL-OCT), a variant of standard OCT, was developed to monitor the chemotherapeutic response of cancers. For example, TL-OCT was used to investigate the therapeutic response of ovarian cancer nodules to cisplatin: a front-line agent for the treatment of ovarian cancer. ${ }^{164}$ A TL-OCT movie showed that the ovarian nodules were initially observed in growth arrest, with no significant structural changes observed during the first $20 \mathrm{~h}$ posttreatment. After one day of therapy, only small vacuoles could be seen appearing in the tumor structures. However, after $40 \mathrm{~h}$ posttreatment, most large ( $>200 \mathrm{~mm}$ in diameter) nodules exhibited substantial changes in their peripheral regions, with smaller nodules being destroyed completely. By $60 \mathrm{~h}$ posttreatment, the peripheral damage to the larger nodules was far more severe, with small nodules disintegrating into multiple smaller fragments. ${ }^{164,165}$

The capability of OCT to monitor a therapeutic response was also demonstrated in a mouse model. ${ }^{166}$ This study imaged mice with MCaIV (murine breast carcinoma) tumors during treatment with vascular endothelial growth factor receptor-2 (VEGFR-2)blocking monoclonal antibody DC101. Control mice with MCaIV tumors received nonspecific rat IgG. With OCT-angiography, a denser and more chaotic vascular network was found in the control group, relative to the treated group [Fig. 11(a)]. The researchers further applied OCT to investigate direct targeting of cancer cells, and found a reduction in both mean intratumor vessel length ( $p=0.001)$ and diameter $(p=0.029)$ with DC101 treatment [Fig. 11(b)]. With diphtheria toxin administered to mice bearing a human colorectal adenocarcinoma xenograft (LS174T) grown in a dorsal skin chamber, apoptosis was evident within 2 days, observed through the associated changes in scattering properties of the tumor [Fig. 11(c)]. During the first $24 \mathrm{~h}$, the tumor volume remained approximately constant [Fig. 11(d)]. After $24 \mathrm{~h}$, the widespread necrotic or apoptotic regions within the volume of the treated tumors were observed, and the mean tumor volume began to decrease rapidly. After $48 \mathrm{~h}$, the intratumor vessel length rapidly decreased, presumably as a result of the downregulation or depletion of tumor cell-derived angiogenic growth factors [Fig. 11(d)]. Another study demonstrated that OCT could be useful for monitoring the use of 5-aminolevulinic acid (ALA) for the photodynamic therapy (PDT) treatment of oral squamous cell carcinoma. ${ }^{167}$ The study found that the boundary between the epithelial (EP) and lamina propria (LP) layers disappeared in the cross-sectional OCT images acquired from the cancerous oral tissues. The boundary between EP and LP layers could not be seen distinctly 1 week after the first ALA-PDT treatment was administered. However, the boundary between EP and LP layers was shown clearly in OCT images acquired one week after the third ALA-PDT treatment, indicating an effective treatment response of the oral cancer to the ALA-PDT. ${ }^{167}$

\section{Insights and Future Directions}

The reported volume of technological advances, preclinical research, and clinical human studies using OCT for oncological imaging is large and diverse, demonstrating the promising potential of OCT for cancer detection and diagnosis, and for the monitoring of cancer therapy. However, somewhat surprisingly, OCT has not been as widely evaluated in larger clinical studies and oncology trials, commercialized for cancer imaging applications, and adopted in clinical oncology as it has in the fields of ophthalmology, cardiology, and gastroenterology. There are several possible reasons for this disparity, despite the significant motivation and effort of many researchers and clinicians, and the potential impact that OCT could have in cancer imaging.

First, technical challenges associated with OCT appear to be more problematic and limiting in oncology, compared with ophthalmology, cardiology, and gastroenterology. For instance, the trade-off between depth of field and transverse resolution is a more significant factor because often cellular and subcellular features are used for cancer diagnostics, and depths of several millimeters are preferred (roughly on the scale of histological sections). Often large areas and volumes of tissue need to be 


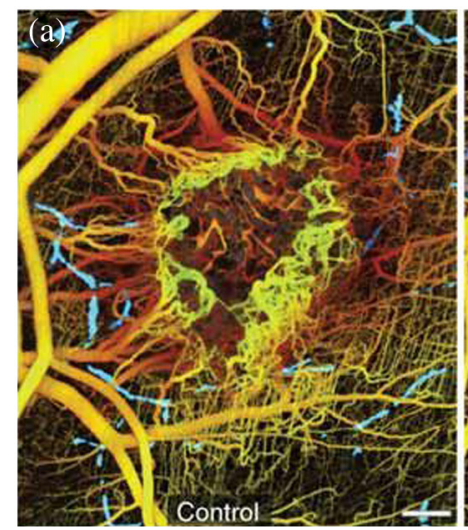

(c)
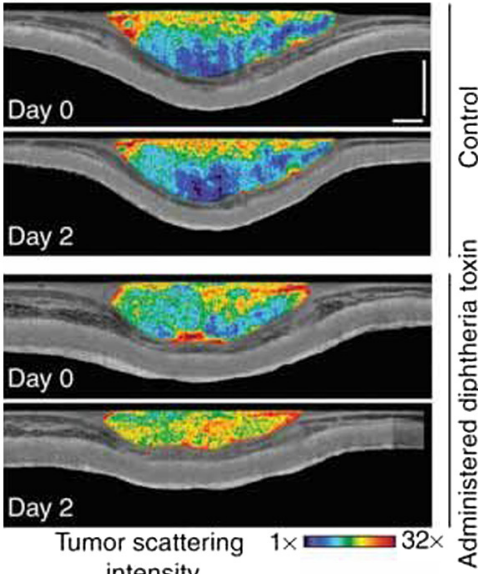

intensity

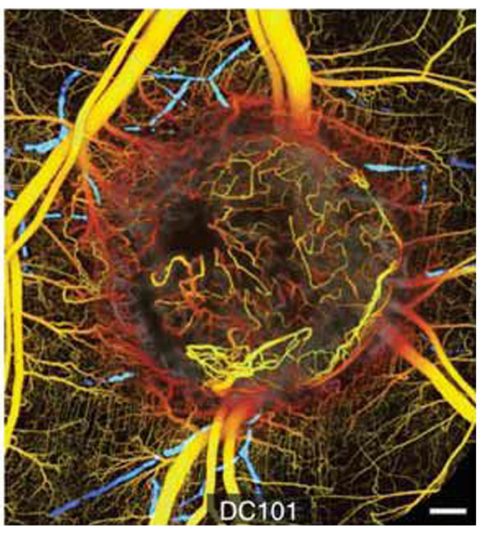

(d)

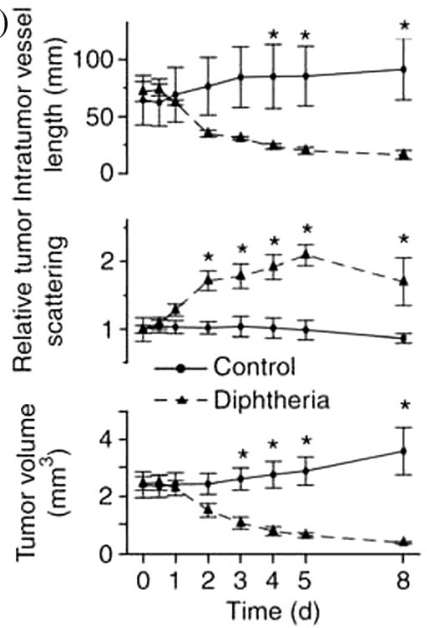

(b)
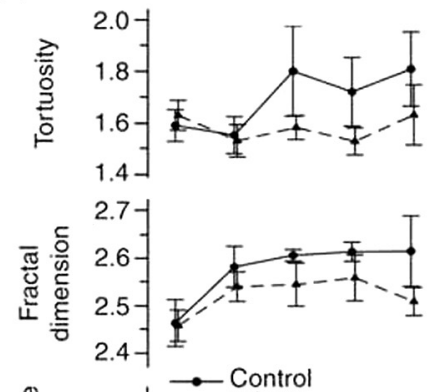

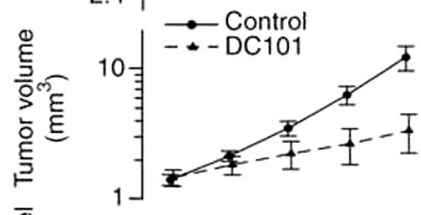
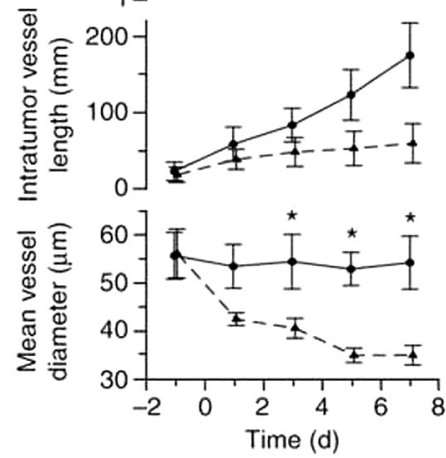

Fig. 11 Multiparametric response of directed anticancer therapy characterized by OCT. (a) OCT images of representative control and treated tumors 5 days after initiation of antiangiogenic VEGFR-2. The lymphatic vascular networks are also presented (blue) for both tumors. (b) Quantification of tumor volume, vascular geometry, and morphology in response to VEGFR-2 blockade. Control, $n=5$; treated, $n=6$. (c) OCT images of tissue scattering immediately before and 2 days after administration of targeted cytotoxic therapy (diphtheria toxin) or saline to mice bearing human tumor xenografts (LS174T) in dorsal skinfold chambers. Apoptosis induced by the diphtheria toxin is manifested as increased tissue scattering relative to control mice. (d) Quantification of the response to diphtheria toxin administration. Control, $n=3$; treated, $n=3$. Scale bars represent $500 \mu \mathrm{m}$. Statistically significant differences $(p<0.05)$ at given time points are denoted by asterisks. Data are presented as mean \pm s.e.m. Figure reprinted with permission from Ref. 166.

imaged at high cellular resolution to detect and diagnose cancer. OCT image interpretation is often more challenging for oncologists, who are more familiar with interpreting planar x-ray, $\mathrm{x}$-ray computed tomography, nuclear imaging, and magnetic resonance images, but not necessarily trained or accustomed to looking for micron-scale changes in images that cover small fields of view, or in images that do not necessarily reveal cellular features that are as familiar as those in histological images. Importantly in oncology, there are no clear technological precedents that have served to make oncologists familiar with and accepting of new and more advanced diagnostic imaging techniques. In contrast, ophthalmologists are inherently familiar with optics and optical imaging instruments in their daily practice, and interventional cardiologists are familiar with and regularly use intravascular ultrasound for imaging the coronary arteries. Perhaps the closest precedent for oncologists is the $\mathrm{H} \& \mathrm{E}$ or immunohistochemically stained histopathology, but the challenge remains to compare a stained histological section to a gray-scale OCT image with each based on different contrast mechanisms and revealing different features.
Second, recognizable cultural differences exist between scientists/engineers and clinicians that potentially hinder the translation of OCT from the laboratory to the clinic. Scientists and engineers are often more focused on driving the technological advances of OCT. However, as a relatively new and nonstandard imaging technology in oncology, oncologists often find it challenging to manipulate an OCT instrument, integrate the use of OCT into patient workflow, and interpret the OCT image data for medical decision making.

Third, larger clinical human trials, including multi-institutional clinical trials, are lacking to statistically and definitively show the true clinical value of OCT in oncology to oncologists, patients, and the general public. Investments in launching larger clinical trials are usually not made unless the technological developments of the new imaging modality have stabilized. As a community, we have appreciated and leveraged the rapid technological advances in OCT over the past several decades; however, these ever-changing imaging platforms have likely delayed the launch of larger clinical trials in oncology. Fortunately, today, with a solid technological foundation, the 
OCT technology is poised to begin these seminal clinical studies and trials.

In the following sections, we have also posed some future directions that may be needed to refine the OCT technology, remove the cultural barriers to the clinical translation of OCT, and show the true clinical value of OCT in oncology.

\subsection{Ultrafast OCT for High-Speed Volumetric Imaging in Oncology}

Real-time volumetric OCT imaging with a large field of view is of great importance in oncology, playing a key role to measure tumor volume, locate tumors anatomically, and define the tumor microenvironment. Traditionally, ultrasound imaging ${ }^{168}$ or microcomputed tomography $(\mu \mathrm{CT}),{ }^{169}$ which have the advantages of large imaging fields and deep penetration, have been used for this purpose. However, the soft-tissue contrast of ultrasound and $\mu \mathrm{CT}$ is suboptimal, imposing limits on their potential for interpretation of the tumor anatomy in 3-D. Volumetric imaging with OCT can be complementary to ultrasound imaging and $\mu \mathrm{CT}$, providing greater contrast and the potential to detect tumor margins in the operating room, as well as better elucidate the microenvironment around the tumor site. Volumetric realtime OCT acquisition and visualization has been demonstrated with a benchtop SS-OCT system enabled by a high-sweep rate Fourier-domain mode-locked laser and graphics processing unit (GPU)-based data processing, ${ }^{170}$ as well as by benchtop SD-OCT facilitated by optical demultiplexers, an FPGA, and a GPU. ${ }^{171}$ Noticeably, commercially available megahertz-rate swept laser sources show great potential for volumetric oncological imaging using OCT. ${ }^{172}$ Nevertheless, the successful application of ultrafast OCT in the operating room requires the development of dedicated flexible or handheld probes compatible with the ultrafast OCT system specifications. Since commercially available MEMS-scanning mirrors that have commonly been embedded in handheld probes have limited scanning rates (on the order of hundreds of $\mathrm{Hz}$ ), more efforts are needed to develop high-speed MEMS-scanning mirrors that can be coupled with ultrafast OCT systems for high-speed volumetric imaging in oncology.

\subsection{Computed Optical Interferometric Tomography for Oncological Imaging}

Using high NA objectives to achieve higher transverse imaging resolution, OCM enables the collection of high-resolution en face images in oncological applications but with a more limited DOF. However, with higher NA optics, the image quality of OCM is more affected by and more sensitive to optical aberrations. Hardware-based methods, and hardware-assisted computational imaging approaches, have been proposed to address the DOF limitation and compensate for optical aberrations. Examples include wavefront engineering for non-Gaussian beam illumination, ${ }^{173-178}$ complex wavefront shaping, ${ }^{179}$ and depth-encoded synthetic apertures ${ }^{180}$ to obtain a large DOF image with high transverse resolution. Hardwarebased adaptive optics ${ }^{181}$ and coherence-gated sensor-less adaptive optics ${ }^{182}$ have been developed for optical aberration compensation. However, the hardware-related methods require special hardware setup configurations in the system. On the other hand, computed imaging techniques including interferometric synthetic aperture microscopy (ISAM) and computational adaptive optics (CAO) have been combined with OCM to overcome this DOF limitation, and the effects of optical aberrations. First demonstrated on ex vivo human breast tissue, ${ }^{23}$ the potential of combined OCM, ISAM, and CAO for highresolution oncological imaging at large DOFs warrants further investigations. Further, the integration of computed optical interferometric tomography methods implemented with ultrafast OCT hardware promises to offer high-resolution, ultrahigh-speed volumetric imaging in oncology.

\subsection{Artificial Intelligence and Machine Learning Algorithms for Cancer Detection Using OCT}

Since specialized expertise is often needed to interpret the oncological OCT images, and this expertise is often costly and/or difficult to obtain in poorly resourced regions, automated robust tissue classification algorithms to differentiate cancer/precancer from normal tissues are highly needed. In the past few years, there has been growing interest in the use of artificial intelligence and machine learning algorithms for analyzing OCT data to achieve automated robust tissue classification. ${ }^{134}$ For instance, a fully automated procedure to detect BCC in ex vivo human skin from PS-OCT images was reported. ${ }^{134} \mathrm{~A}$ total of 1314 features (613 from intensity data and 701 from polarization retardation data) were extracted from both intensity and polarization images, followed by the use of a support vector machine with Gaussian kernel, forward search algorithm and leave-one-patient-out-cross-validation, achieving an accuracy, sensitivity, and specificity all around $95 \% .{ }^{134}$ Considering the automated, robust, and powerful tissue diagnosis that can potentially be achieved by artificial intelligence and machine learning algorithms, it is expected that these will play incredibly important roles in the future for OCT imaging in the field of oncology.

\subsection{Contrast Agents for Site-Specific Targeting and Image Enhancement in OCT}

Contrast agents that produce specific image signatures have been utilized in virtually every imaging modality, including ultrasound, ${ }^{183} \mathrm{x}$-ray CT, ${ }^{184} \mathrm{MRI},{ }^{185}$ and optical microscopy. ${ }^{186}$ It is, therefore, not surprising that a variety of different contrast agents have been developed for OCT. Examples include magnetic nanoparticles or microspheres that can be detected with magnetomotive-OCT, ${ }^{187}$ encapsulating protein-shell oil-filled microspheres, which contain scattering nanoparticles within the shell or core, ${ }^{188}$ gold nanorods, nanospheres, or nanocages that have tuned plasmon resonances, and near-infrared dyes, that can be targeted to specific cells or tissue types, for enhanced scattering in OCT or for spectroscopic OCT, ${ }^{189-191}$ as well as gold nanospheres and nanorods, that can be heated in a modulated manner via incident optical energy for photothermal OCT. ${ }^{192}$ With the ability for site-specific molecular targeting and imaging enhancements, contrast agents will continue to be a future direction for OCT imaging. Regulatory processes will be important for safety approvals but may also slow down the clinical translation and use of contrast agents for oncological imaging with OCT.

\subsection{Integrated Optical Molecular Imaging Modalities with OCT for Oncological Imaging}

The nature of the information provided by OCT and optical molecular imaging modalities (structural versus biochemical) creates the possibility that their combination may be more 
sensitive to early phenotypic changes than the established oncological imaging modalities or either modality alone. ${ }^{193-195}$ Preliminary work that integrates OCT with either fluorescence spectroscopy or Raman spectroscopy has demonstrated their potential for enhanced tissue characterization and oncological imaging. ${ }^{193-195}$ A dual-modal device capable of sequential acquisition of Raman spectroscopy and OCT along a common optical axis was developed. ${ }^{194}$ The device enhances application of both Raman spectroscopy and OCT by precisely guiding Raman spectra acquisition with OCT images while also compensating for the lack of molecular specificity in OCT with the biochemical specificity of Raman spectroscopy. ${ }^{194}$ In addition, a side-viewing Raman spectroscopy-OCT probe was also developed to facilitate oncological imaging. ${ }^{195}$ The potential of integrated OCT and optical molecular imaging modalities for enhancing oncological imaging in humans in vivo warrants further investigations.

\subsection{Multi-Institutional Clinical Trials Involving Interdisciplinary Teams}

Investigator-initiated interdisciplinary clinical trials and coordinated validation at other multi-institutional clinical sites could greatly promote the translation of OCT technology into oncology. Since the multi-institutional clinical trials bring together professionals (i.e., collaborating academic and clinic employee, and research coordinator) from different disciplines, creating a forum where different perspectives can be expressed, good ideas can be put forward, and finally consensus can be obtained to establish the standard operating procedures of OCT instruments, add OCT to the current workflow of the oncologists, and build up the atlas of OCT oncological imaging. Further, multi-institutional clinical trials will allow OCT to be implemented either as a stand-alone imaging modality or in combination with current established oncological imaging modalities on a relatively large patient database with sufficient number for each cancer type and/or subtype, enabling a systematic comparison (i.e., diagnostic accuracy, diagnostic yield, and cost effectiveness) of using OCT and standard imaging modalities for cancer management.

\section{Conclusions}

This paper has provided a broad overview of the technological developments of OCT along with a historical and current review of its application for in vitro, ex vivo, and in vivo cancer diagnostics and for the monitoring of cancer therapy. The application of OCT technology in oncology has evolved over many years of effort by many research groups, individuals, physicians, and companies to translate the technology from the bench to the bedside, and back again, with impressive results. With high diagnostic sensitivity and specificity for the detection of cancer, OCT-based systems are now being developed and commercialized for the demanding clinical applications in medical and surgical oncology, and the clinical settings in which they will be used. The ensuing transformation of medicine and surgery, with improvements in patient health and outcomes, will undoubtedly be the most exciting area for future exploration.

\section{Disclosures}

Stephen Boppart is cofounder and chief medical officer of Diagnostic Photonics, Inc., Chicago, Illinois, which is developing and commercializing interferometric synthetic aperture microscopy for applications in intraoperative tumor margin detection. He also receives royalties for patents licensed by the Massachusetts Institute of Technology related to OCT. Other authors have no potential conflicts of interest to disclose.

\section{Acknowledgments}

We wish to thank all of our colleagues, researchers, and students who have joined us in the pursuit to fundamentally understand the complex process of carcinogenesis, as well as to improve the lives of cancer patients by innovating, developing, and applying the OCT technology. While we have attempted to capture the enormous breadth and depth of research in this area, we apologize that we were not able to fully represent all the impactful papers and results in this continually growing field. Funding to research and write this review was provided in part by the National Institutes of Health (R01 CA213149 and R01 EB023232). Additional information can be found at: http:// biophotonics.illinois.edu.

\section{References}

1. D. G. Bragg, "State-of-the-art assessment. Diagnostic oncologic imaging," Cancer 64(S1), 261-265 (1989).

2. J. Czernin, M. Allen-Auerbach, and H. R. Schelbert, "Improvements in cancer staging with PET/CT: literature-based evidence as of September 2006," J. Nucl. Med. 48(1 suppl), 78S-88S (2007).

3. J. V. Frangioni, "New technologies for human cancer imaging," J. Clin. Oncol. 26(24), 4012-4021 (2008).

4. A. M. Zysk et al., "Optical coherence tomography: a review of clinical development from bench to bedside," J. Biomed. Opt. 12(5), 051403 (2007).

5. R. Choe et al., "Diffuse optical tomography of breast cancer during neoadjuvant chemotherapy: a case study with comparison to MRI," Med. Phys. 32(4), 1128-1139 (2005).

6. National Institute of Biomedical Imaging and Bioengineering, "Optical imaging fact sheet," 2016, https://www.nibib.nih.gov/scienceeducation/science-topics/optical-imaging.

7. D. Huang et al., "Optical coherence tomography," Science 254(5035), 1178-1181 (1991).

8. C. A. Jesser et al., "High resolution imaging of transitional cell carcinoma with optical coherence tomography: feasibility for the evaluation of bladder pathology," Br. J. Radiol. 72(864), 1170-1176 (1999).

9. S. Brand et al., "Optical coherence tomography in the gastrointestinal tract," Endoscopy 32(10), 796-803 (2000).

10. A. V. D'Amico et al., "Optical coherence tomography as a method for identifying benign and malignant microscopic structures in the prostate gland," Urology 55(5), 783-787 (2000).

11. N. D. Gladkova et al., "In vivo optical coherence tomography imaging of human skin: norm and pathology," Skin Res. Technol. 6(1), 6-16 (2000).

12. P. F. Escobar et al., "Diagnostic efficacy of optical coherence tomography in the management of preinvasive and invasive cancer of uterine cervix and vulva," Int. J. Gynecol. Cancer. 14(3), 470-474 (2004).

13. J. P. Williamson et al., "Using optical coherence tomography to improve diagnostic and therapeutic bronchoscopy," Chest 136(1), 272-276 (2009).

14. C. S. Kim et al., "Enhanced detection of early-stage oral cancer in vivo by optical coherence tomography using multimodal delivery of gold nanoparticles," J. Biomed. Opt. 14(3), 034008 (2009).

15. S. J. Erickson-Bhatt et al., "Real-time imaging of the resection bed using a handheld probe to reduce incidence of microscopic positive margins in cancer surgery," Cancer Res. 75(18), 3706-3712 (2015).

16. C. Kut et al., "Detection of human brain cancer infiltration ex vivo and in vivo using quantitative optical coherence tomography," Sci. Transl. Med. 7(292), 292ra100 (2015).

17. M. R. Hee et al., "Quantitative assessment of macular edema with optical coherence tomography," Arch. Ophthalmol. 113(8), 1019-1029 (1995).

18. J. S. Schuman et al., Optical Coherence Tomography of Ocular Diseases, Slack, New Jersey (2004). 
19. M. E. Brezinski and J. G. Fujimoto, "Optical coherence tomography: high-resolution imaging in nontransparent tissue," IEEE J. Sel. Top. Quantum Electron. 5(4), 1185-1192 (1999).

20. Y.-Z. Liu et al., "Computational optical coherence tomography," Biomed. Opt. Express 8(3), 1549-1574 (2017).

21. J. V. Frangioni, "In vivo near-infrared fluorescence imaging," Curr. Opin. Chem. Biol. 7(5), 626-634 (2003).

22. W. Tan et al., "Optical coherence tomography of cell dynamics in three-dimensional tissue models," Opt. Express 14(16), 7159-7171 (2006).

23. Y.-Z. Liu et al., "Computed optical interferometric tomography for high-speed volumetric cellular imaging," Biomed. Opt. Express 5(9), 2988-3000 (2014).

24. R. K. Chhetri et al., "Longitudinal study of mammary epithelial and fibroblast co-cultures using optical coherence tomography reveals morphological hallmarks of pre-malignancy," PloS One 7(11), e49148 (2012).

25. G. Farhat et al., "Detecting apoptosis using dynamic light scattering with optical coherence tomography," J. Biomed. Opt. 16(7), 070505 (2011).

26. W. J. Choi et al., "Full-field optical coherence microscopy for identifying live cancer cells by quantitative measurement of refractive index distribution," Opt. Express 18(22), 23285-23295 (2010).

27. S. A. Boppart et al., "Optical coherence tomography: feasibility for basic research and image-guided surgery of breast cancer," Breast Cancer Res. Treat. 84(2), 85-97 (2004).

28. W. Luo et al., "Optical biopsy of lymph node morphology using optical coherence tomography," Technol. Cancer Res. Treat. 4(5), 539-547 (2005).

29. N. V. Iftimia et al., "A portable, low coherence interferometry based instrument for fine needle aspiration biopsy guidance," Rev. Sci. Instrum. 76(6), 064301 (2005).

30. B. D. Goldberg et al., "Automated algorithm for differentiation of human breast tissue using low coherence interferometry for fine needle aspiration biopsy guidance," J. Biomed. Opt. 13(1), 014014 (2008).

31. A. M. Zysk et al., "Clinical feasibility of microscopically-guided breast needle biopsy using a fiber-optic probe with computer-aided detection," Technol. Cancer Res. Treat. 8(5), 315-321 (2009).

32. N. V. Iftimia et al., "Spectral-domain low coherence interferometry/ optical coherence tomography system for fine needle breast biopsy guidance," Rev. Sci. Instrum. 80(2), 024302 (2009).

33. R. A. McLaughlin et al., "Imaging of breast cancer with optical coherence tomography needle probes: feasibility and initial results," IEEE J. Sel. Top. Quantum Electron. 18(3), 1184-1191 (2012).

34. A. Curatolo et al., "Ultrasound-guided optical coherence tomography needle probe for the assessment of breast cancer tumor margins," Am. J. Roentgenol. 199(4), W520-W522 (2012).

35. K. M. Kennedy et al., "Needle optical coherence elastography for the measurement of microscale mechanical contrast deep within human breast tissues," J. Biomed. Opt. 18(12), 121510 (2013).

36. M. Villiger et al., "Deep tissue volume imaging of birefringence through fibre-optic needle probes for the delineation of breast tumour," Sci. Rep. 6, 28771 (2016).

37. S. A. McLaughlin et al., "Prevalence of lymphedema in women with breast cancer 5 years after sentinel lymph node biopsy or axillary dissection: objective measurements," J. Clin. Oncol. 26(32), 5213-5219 (2008).

38. A. M. Zysk and S. A. Boppart, "Computational methods for analysis of human breast tumor tissue in optical coherence tomography images," J. Biomed. Opt. 11(5), 054015 (2006).

39. P.-L. Hsiung et al., "Benign and malignant lesions in the human breast depicted with ultrahigh resolution and three-dimensional optical coherence tomography," Radiology 244(3), 865-874 (2007).

40. C. Zhou et al., "Integrated optical coherence tomography and microscopy for ex vivo multiscale evaluation of human breast tissues," Cancer Res. 70(24), 10071-10079 (2010).

41. O. Assayag et al., "Large field, high resolution full-field optical coherence tomography: a pre-clinical study of human breast tissue and cancer assessment," Technol. Cancer Res. Treat. 13(5), 455-468 (2014).

42. D. Savastru et al., "Detection of breast surgical margins with optical coherence tomography imaging: a concept evaluation study," J. Biomed. Opt. 19(5), 056001 (2014).
43. H.-J. Ko et al., "Optical coherence elastography of engineered and developing tissue," Tissue Eng. 12(1), 63-73 (2006).

44. X. Liang et al., "Acoustomotive optical coherence elastography for measuring material mechanical properties," Opt. Lett. 34(19), 28942896 (2009).

45. X. Liang et al., "Dynamic spectral-domain optical coherence elastography for tissue characterization," Opt. Express 18(13), 14183-14190 (2010).

46. A. Srivastava et al., "Determination of elastic properties of resected human breast tissue samples using optical coherence tomographic elastography," Strain 47(1), 75-87 (2011).

47. P.-C. Huang et al., "Magnetomotive optical coherence elastography for magnetic hyperthermia dosimetry based on dynamic tissue biomechanics," IEEE J. Sel. Top. Quantum Electron. 22(4), 104-119 (2016).

48. Y. Verma et al., "Imaging of human breast tissue using polarization sensitive optical coherence tomography," Laser Phys. 21(12), 21432148 (2011).

49. F. A. South et al., "Differentiation of ex vivo human breast tissue using polarization-sensitive optical coherence tomography," Biomed. Opt. Express 5(10), 3417-3426 (2014).

50. B. F. Kennedy et al., "Optical coherence micro-elastography: mechanical-contrast imaging of tissue microstructure," Biomed. Opt. Express 5(7), 2113-2124 (2014).

51. B. F. Kennedy et al., "Investigation of optical coherence microelastography as a method to visualize cancers in human breast tissue," Cancer Res. 75(16), 3236-3245 (2015).

52. C. Zhou et al., "Photothermal optical coherence tomography in ex vivo human breast tissues using gold nanoshells," Opt. Lett. 35(5), 700-702 (2010).

53. F. T. Nguyen et al., "Optical coherence tomography: the intraoperative assessment of lymph nodes in breast cancer," IEEE Eng. Med. Biol. Mag. 29(2), 63-70 (2010).

54. R. A. McLaughlin et al., "Imaging of human lymph nodes using optical coherence tomography: potential for staging cancer," Cancer Res. 70(7), 2579-2584 (2010).

55. R. John et al., "Three-dimensional optical coherence tomography for optical biopsy of lymph nodes and assessment of metastatic disease," Ann. Surg. Oncol. 20(11), 3685-3693 (2013).

56. R. A. McLaughlin et al., "Parametric imaging of cancer with optical coherence tomography," J. Biomed. Opt. 15(4), 046029 (2010).

57. L. Scolaro et al., "Parametric imaging of the local attenuation coefficient in human axillary lymph nodes assessed using optical coherence tomography," Biomed. Opt. Express 3(2), 366-379 (2012).

58. J. G. Sun et al., "Segmentation and correlation of optical coherence tomography and X-ray images for breast cancer diagnostics," J. Innov. Opt. Health. Sci. 6(02), 1350015 (2013).

59. A. C. Sullivan, J. P. Hunt, and A. L. Oldenburg, "Fractal analysis for classification of breast carcinoma in optical coherence tomography," J. Biomed. Opt. 16(6), 066010 (2011).

60. M. Mujat et al., "Automated algorithm for breast tissue differentiation in optical coherence tomography," J. Biomed. Opt. 14(3), 034040 (2009).

61. S. A. Boppart et al., "Optical coherence tomography for neurosurgical imaging of human intracortical melanoma," Neurosurgery 43(4), 834-841 (1998).

62. S. A. Boppart, "Optical coherence tomography: technology and applications for neuroimaging," Psychophysiology 40(4), 529-541 (2003).

63. K. Bizheva et al., "Imaging ex vivo and in vitro brain morphology in animal models with ultrahigh resolution optical coherence tomography," J. Biomed. Opt. 9(4), 719-724 (2004).

64. K. Bizheva et al., "Imaging ex vivo healthy and pathological human brain tissue with ultra-high-resolution optical coherence tomography," J. Biomed. Opt. 10(1), 011006 (2005).

65. H. J. Böhringer et al., "Optical coherence tomography for experimental neuroendoscopy," Minim. Invasive Neurosurg. 49(05), 269-275 (2006).

66. H. J. Böhringer et al., "Time-domain and spectral-domain optical coherence tomography in the analysis of brain tumor tissue," Laser Surg. Med 38(6), 588-597 (2006).

67. H. J. Böhringer et al., "Imaging of human brain tumor tissue by near-infrared laser coherence tomography," Acta Neurochirurgica 151(5), 507-517 (2009). 
68. M. Finke et al., "Automatic scanning of large tissue areas in neurosurgery using optical coherence tomography," Int. J. Med. Robot. 8(3), 327-336 (2012).

69. J. Sun et al., "Refractive index measurement of acute rat brain tissue slices using optical coherence tomography," Opt. Express 20(2), 10841095 (2012).

70. O. Assayag et al., "Imaging of non-tumorous and tumorous human brain tissues with full-field optical coherence tomography," NeuroImage: Clin. 2, 549-557 (2013).

71. R. U. Maheswari et al., "Implementation of optical coherence tomography (OCT) in visualization of functional structures of cat visual cortex," Opt. Commun. 202(1), 47-54 (2002).

72. S. R. Kantelhardt et al., "Evaluation of a completely robotized neurosurgical operating microscope," Neurosurgery 72(suppl_1), A19-A26 (2013).

73. M. Tsuboi et al., "Optical coherence tomography in the diagnosis of bronchial lesions," Lung Cancer 49(3), 387-394 (2005).

74. L. P. Hariri et al., "Volumetric optical frequency domain imaging of pulmonary pathology with precise correlation to histopathology," Chest 143(1), 64-74 (2013).

75. L. P. Hariri et al., "Optical frequency domain imaging of ex vivo pulmonary resection specimens: obtaining one to one image to histopathology correlation," J. Vis. Exp. 71, 3855 (2013).

76. L. P. Hariri et al., "Diagnosing lung carcinomas with optical coherence tomography," Ann. Am. Thorac. Soc. 12(2), 193-201 (2015).

77. L. P. Hariri et al., "Seeing beyond the bronchoscope to increase the diagnostic yield of bronchoscopic biopsy," Am. J. Respir. Crit. Care Med. 187(2), 125-129 (2013).

78. N. Hanna et al., "Two-dimensional and 3-dimensional optical coherence tomographic imaging of the airway, lung, and pleura," J. Thorac. Cardiovasc. Surg. 129(3), 615-622 (2005).

79. S. C. Whiteman et al., "Optical coherence tomography: real-time imaging of bronchial airways microstructure and detection of inflammatory/neoplastic morphologic changes," Clin. Cancer Res. 12(3), 813-818 (2006).

80. M. Jain et al., "Full-field optical coherence tomography for the analysis of fresh unstained human lobectomy specimens," J. Pathol. Inform. 4, 26 (2013).

81. J. J. Armstrong et al., "In vivo size and shape measurement of the human upper airway using endoscopic long-range optical coherence tomography," Opt. Express 11(15), 1817-1826 (2003).

82. M. S. Leigh et al., "Anatomical optical coherence tomography for long-term, portable, quantitative endoscopy," IEEE Trans. Biomed. Eng. 55(4), 1438-1446 (2008).

83. R. A. McLaughlin et al., "Applying anatomical optical coherence tomography to quantitative 3D imaging of the lower airway," Opt. Express 16(22), 17521-17529 (2008).

84. J. P. Williamson et al., "Measuring airway dimensions during bronchoscopy using anatomical optical coherence tomography," Eur. Respir. J. 35(1), 34-41 (2010).

85. J. P. Williamson et al., "Elastic properties of the central airways in obstructive lung diseases measured using anatomical optical coherence tomography," Am. J. Respir. Crit. Care Med. 183(5), 612-619 (2011).

86. B. C. Quirk et al., "In situ imaging of lung alveoli with an optical coherence tomography needle probe," J. Biomed. Opt. 16(3), 036009 (2011).

87. S. Lam et al., "In vivo optical coherence tomography imaging of preinvasive bronchial lesions," Clin. Cancer Res. 14(7), 2006-2011 (2008).

88. L. A. Torre et al., "Global cancer statistics, 2012," CA Cancer J. Clin. 65(2), 87-108 (2015).

89. C. Pitris et al., "Feasibility of optical coherence tomography for highresolution imaging of human gastrointestinal tract malignancies," J. Gastroenterol. 35(2), 87-92 (2000).

90. J. A. Izatt et al., "Optical coherence tomography and microscopy in gastrointestinal tissues," IEEE J. Sel. Top. Quantum Electron. 2(4), 1017-1028 (1996).

91. K. Kobayashi et al., "High-resolution cross-sectional imaging of the gastrointestinal tract using optical coherence tomography: preliminary results," Gastrointest. Endosc. 47(6), 515-523 (1998).

92. J. M. Poneros et al., "Diagnosis of specialized intestinal metaplasia by optical coherence tomography," Gastroenterology 120(1), 7-12 (2001).
93. P.-L. Hsiung et al., "Ultrahigh-resolution and 3-dimensional optical coherence tomography ex vivo imaging of the large and small intestines," Gastrointest. Endosc. 62(4), 561-574 (2005).

94. A. D. Aguirre et al., "Cellular resolution ex vivo imaging of gastrointestinal tissues with optical coherence microscopy," J. Biomed. Opt. 15(1), 016025 (2010).

95. L. P. Hariri et al., "EX vivo optical coherence tomography and laserinduced fluorescence spectroscopy imaging of murine gastrointestinal tract," Comp. Med. 57(2), 175-185 (2007).

96. N. Iftimia et al., "Fluorescence-guided optical coherence tomography imaging for colon cancer screening: a preliminary mouse study," Biomed. Opt. Express 3(1), 178-191 (2012).

97. G. J. Tearney et al., "In vivo endoscopic optical biopsy with optical coherence tomography," Science 276(5321), 2037-2039 (1997).

98. G. J. Tearney et al., "Optical biopsy in human gastrointestinal tissue using optical coherence tomography," Am. J. Gastroenterol. 92(10), 1800-1804 (1997).

99. S. Jäckle et al., "In vivo endoscopic optical coherence tomography of the human gastrointestinal tract-toward optical biopsy," Endoscopy 32(10), 743-749 (2000).

100. M. V. Sivak et al., "High-resolution endoscopic imaging of the GI tract using optical coherence tomography," Gastrointest. Endosc. 51(4), 474-479 (2000).

101. J. A. Evans et al., "Optical coherence tomography to identify intramucosal carcinoma and high-grade dysplasia in Barrett's esophagus," Clin. Gastroenterol. Hepatol. 4(1), 38-43 (2006).

102. B. J. Vakoc et al., "Comprehensive esophageal microscopy by using optical frequency-domain imaging (with video)," Gastrointest. Endosc. 65(6), 898-905 (2007).

103. J. Xi et al., "High-resolution OCT balloon imaging catheter with astigmatism correction," Opt. Lett. 34(13), 1943-1945 (2009).

104. W. Kang et al., "Endoscopically guided spectral-domain OCT with double-balloon catheters," Opt. Express 18(16), 17364-17372 (2010).

105. A. R. Tumlinson et al., "Endoscope-tip interferometer for ultrahighresolution frequency domain optical coherencetomography in mouse colon," Opt. Express 14(5), 1878-1887 (2006).

106. A. Das et al., "High-resolution endoscopic imaging of the GI tract: a comparative study of optical coherence tomography versus highfrequency catheter probe EUS," Gastrointest. Endosc. 54(2), 219-224 (2001).

107. M. J. Gora et al., "Tethered capsule endomicroscopy enables less invasive imaging of gastrointestinal tract microstructure," Nat. Med. 19(2), 238-240 (2013).

108. G. J. Ughi et al., "Automated segmentation and characterization of esophageal wall in vivo by tethered capsule optical coherence tomography endomicroscopy," Biomed. Opt. Express 7(2), 409-419 (2016).

109. V. X. D. Yang et al., "High speed, wide velocity dynamic range Doppler optical coherence tomography (Part III): in vivo endoscopic imaging of blood flow in the rat and human gastrointestinal tracts," Opt. Express 11(19), 2416-2424 (2003).

110. V. X. D. Yang et al., "Endoscopic Doppler optical coherence tomography in the human GI tract: initial experience," Gastrointest. Endosc. 61(7), 879-890 (2005).

111. S. P. Lerner et al., "Optical coherence tomography as an adjunct to white light cystoscopy for intravesical real-time imaging and staging of bladder cancer," Urology 72(1), 133-137 (2008).

112. G. J. Tearney et al., "Optical biopsy in human urologic tissue using optical coherence tomography," J. Urol. 157(5), 1915-1919 (1997).

113. Y. Pan et al., "Detection of tumorigenesis in rat bladders with optical coherence tomography," Med. Phys. 28(12), 2432-2440 (2001).

114. Z. Yuan et al., "High-resolution imaging diagnosis and staging of bladder cancer: comparison between optical coherence tomography and high-frequency ultrasound," J. Biomed. Opt. 13(5), 054007 (2008).

115. Z. Yuan et al., "On the possibility of time-lapse ultrahigh-resolution optical coherence tomography for bladder cancer grading," $J$. Biomed. Opt. 14(5), 050502 (2009).

116. D. Daniltchenko et al., "Utilizing optical coherence tomography (OCT) for visualization of urothelial diseases of the urinary bladder," Der. Radiologe 46(7), 584-589 (2006).

117. B. Hermes et al., "Visualization of the basement membrane zone of the bladder by optical coherence tomography: feasibility of noninvasive evaluation of tumor invasion," Urology 72(3), 677-681 (2008). 
118. F. I. Feldchtein et al., "Endoscopic applications of optical coherence tomography," Opt. Express 3(6), 257-270 (1998).

119. H. Ren et al., "Diagnosis of bladder cancer with microelectromechanical systems-based cystoscopic optical coherence tomography," Urology 74(6), 1351-1357 (2009).

120. Z. Wang et al., "In vivo bladder imaging with microelectromechanicalsystems-based endoscopic spectral domain optical coherence tomography," J. Biomed. Opt. 12(3), 034009 (2007).

121. C. A. Lingley-Papadopoulos et al., "Computer recognition of cancer in the urinary bladder using optical coherence tomography and texture analysis," J. Biomed. Opt. 13(2), 024003 (2008).

122. A. Karl et al., "Optical coherence tomography for bladder cancer-ready as a surrogate for optical biopsy?-results of a prospective mono-centre study," Eur. J. Med. Res. 15(3), 131 (2010).

123. Y. T. Pan et al., "Enhancing early bladder cancer detection with fluorescence-guided endoscopic optical coherent tomography," Opt. Lett. 28(24), 2485-2487 (2003).

124. Z. G. Wang et al., "Fluorescence guided optical coherence tomography for the diagnosis of early bladder cancer in a rat model," J. Urol. 174(6), 2376-2381 (2005).

125. J. Schmidbauer et al., "Fluorescence cystoscopy with high-resolution optical coherence tomography imaging as an adjunct reduces falsepositive findings in the diagnosis of urothelial carcinoma of the bladder," Eur. Urol. 56(6), 914-919 (2009).

126. E. Kiseleva et al., "Differential diagnosis of human bladder mucosa pathologies in vivo with cross-polarization optical coherence tomography," Biomed. Opt. Express 6(4), 1464-1476 (2015).

127. N. Gladkova et al., "Cross-polarization optical coherence tomography for early bladder-cancer detection: statistical study," J. Biophotonics 4(7-8), 519-532 (2011).

128. E. B. Russell, P. R. Carrington, and B. R. Smoller, "Basal cell carcinoma: a comparison of shave biopsy versus punch biopsy techniques in subtype diagnosis," J. Am. Acad. Dermatol. 41(1), 69-71 (1999).

129. E. Sattler, R. Kästle, and J. Welzel, "Optical coherence tomography in dermatology," J. Biomed. Opt. 18(6), 061224 (2013).

130. T. Gambichler et al., "In vivo optical coherence tomography of basal cell carcinoma," J. Dermatol. Sci. 45(3), 167-173 (2007).

131. V. R. Korde et al., "Using optical coherence tomography to evaluate skin sun damage and precancer," Laser Surg. Med 39(9), 687-695 (2007).

132. J. Strasswimmer et al., "Polarization-sensitive optical coherence tomography of invasive basal cell carcinoma," J. Biomed. Opt. 9(2), 292-298 (2004).

133. M. Mogensen et al., "Assessment of optical coherence tomography imaging in the diagnosis of non-melanoma skin cancer and benign lesions versus normal skin: observer-blinded evaluation by dermatologists and pathologists," Dermatol. Surg. 35(6), 965-972 (2009).

134. T. Marvdashti et al., "Classification of basal cell carcinoma in human skin using machine learning and quantitative features captured by polarization sensitive optical coherence tomography," Biomed. Opt. Express 7(9), 3721-3735 (2016).

135. C. Rivera, "Essentials of oral cancer," Int. J. Clin. Exp. Pathol. 8(9), 11884 (2015).

136. S. Tiziani, V. Lopes, and U. L. Günther, "Early stage diagnosis of oral cancer using 1 H NMR-based metabolomics," Neoplasia 11(3), 269 (2009).

137. W. Jerjes et al., "In vitro examination of suspicious oral lesions using optical coherence tomography," Br. J. Oral Maxillofac. Surg. 48(1), 18-25 (2010).

138. Z. Hamdoon et al., "Optical coherence tomography in the assessment of suspicious oral lesions: an immediate ex vivo study," Photodiagnosis Photodyn. Ther. 10(1), 17-27 (2013).

139. M.-T. Tsai et al., "Differentiating oral lesions in different carcinogenesis stages with optical coherence tomography," J. Biomed. Opt. 14(4), 044028 (2009).

140. P. Wilder-Smith et al., "In vivo diagnosis of oral dysplasia and malignancy using optical coherence tomography: preliminary studies in 50 patients," Laser Surg. Med. 41(5), 353-357 (2009).

141. A. M. D. Lee et al., "Wide-field in vivo oral OCT imaging," Biomed. Opt. Express 6(7), 2664-2674 (2015).

142. A. V. Shakhov et al., "Optical coherence tomography monitoring for laser surgery of laryngeal carcinoma," J. Surg. Oncol. 77(4), 253-258 (2001).
143. B. J. F. Wong et al., "In vivo optical coherence tomography of the human larynx: normative and benign pathology in 82 patients," Laryngoscope 115(11), 1904-1911 (2005).

144. W. B. Armstrong et al., "Optical coherence tomography of laryngeal cancer," Laryngoscope 116(7), 1107-1113 (2006).

145. M. Kraft et al., "Technique of optical coherence tomography of the larynx during microlaryngoscopy," Laryngoscope 117(5), 950-952 (2007).

146. M. Kraft et al., "Clinical value of optical coherence tomography in laryngology," Head Neck 30(12), 1628-1635 (2008).

147. A. Sepehr et al., "Optical coherence tomography of the larynx in the awake patient," Otolaryngol. Head Neck Surg. 138(4), 425-429 (2008).

148. T. Just et al., "Optical coherence tomography allows for the reliable identification of laryngeal epithelial dysplasia and for precise biopsy: a clinicopathological study of 61 patients undergoing microlaryngoscopy," Laryngoscope 120(10), 1964-1970 (2010).

149. J. A. Burns et al., "Polarization-sensitive optical coherence tomography imaging of benign and malignant laryngeal lesions: an in vivo study," Otolaryngol. Head Neck Surg. 145(1), 91-99 (2011).

150. M. Löning et al., "Optische Kohärenztomographie-ein neues hochauflösendes Schnittbildverfahren als Ergänzung zur Kolposkopie," Geburtshilfe Frauenheilkd. 63(11), 1158-1161 (2003).

151. J. K. Gallwas et al., "Optical coherence tomography for the diagnosis of cervical intraepithelial neoplasia," Laser Surg. Med 43(3), 206-212 (2011).

152. P. F. Escobar et al., "Optical coherence tomography as a diagnostic aid to visual inspection and colposcopy for preinvasive and invasive cancer of the uterine cervix," Int. J. Gynecol. Cancer. 16(5), 18151822 (2006).

153. A. F. Zuluaga et al., "Optical coherence tomography: a pilot study of a new imaging technique for noninvasive examination of cervical tissue," Am. J. Obstet. Gynecol. 193(1), 83-88 (2005).

154. J. Gallwas et al., "Optical coherence tomography as a non-invasive imaging technique for preinvasive and invasive neoplasia of the uterine cervix," Ultrasound Obstet. Gynecol. 36(5), 624-629 (2010).

155. W. Kang et al., "Diagnostic efficacy of computer extracted image features in optical coherence tomography of the precancerous cervix," Med. Phys. 38(1), 107-113 (2011).

156. C. Pitris et al., "High-resolution imaging of gynecologic neoplasms using optical coherence tomography," Obstet. Gynecol. 93(1), 135-139 (1999).

157. D. W. Siemann, D. J. Chaplin, and M. R. Horsman, "Vascular-targeting therapies for treatment of malignant disease," Cancer 100(12), 2491-2499 (2004).

158. M. Arruebo et al., "Assessment of the evolution of cancer treatment therapies," Cancers 3(3), 3279-3330 (2011).

159. I. Rizvi et al., "Synergistic enhancement of carboplatin efficacy with photodynamic therapy in a three-dimensional model for micrometastatic ovarian cancer," Cancer Res. 70(22), 9319-9328 (2010).

160. O. J. Klein, Y. K. Jung, and C. L. Evans, "Longitudinal, quantitative monitoring of therapeutic response in $3 \mathrm{D}$ in vitro tumor models with OCT for high-content therapeutic screening," Methods 66(2), 299-311 (2014).

161. Y. Jung et al., "Longitudinal, label-free, quantitative tracking of cell death and viability in a 3D tumor model with OCT," Sci. Rep. 6, 27017 (2016).

162. F. J. van der Meer et al., "Apoptosis-and necrosis-induced changes in light attenuation measured by optical coherence tomography," Lasers Med. Sci. 25(2), 259-267 (2010).

163. Y. Zhao et al., "Longitudinal label-free tracking of cell death dynamics in living engineered human skin tissue with a multimodal microscope," Biomed. Opt. Express 5(10), 3699-3716 (2014).

164. C. L. Evans et al., "In vitro ovarian tumor growth and treatment response dynamics visualized with time-lapse OCT imaging," Opt. Express 17(11), 8892-8906 (2009).

165. Y. Jung et al., "Label-free, longitudinal visualization of PDT response in vitro with optical coherence tomography," Isr. J. Chem. 52(8-9), 728-744 (2012).

166. B. J. Vakoc et al., "Three-dimensional microscopy of the tumor microenvironment in vivo using optical frequency domain imaging," Nat. Med. 15(10), 1219-1223 (2009). 
167. H.-C. Wang, M.-T. Tsai, and C.-P. Chiang, "Visual perception enhancement for detection of cancerous oral tissue by multi-spectral imaging," J. Opt. 15(5), 055301 (2013).

168. M. A. Hammoud et al., "Use of intraoperative ultrasound for localizing tumors and determining the extent of resection: a comparative study with magnetic resonance imaging," J. Neurosurg. 84(5), 737-741 (1996).

169. A. A. Folarin et al., "Three-dimensional analysis of tumour vascular corrosion casts using stereoimaging and micro-computed tomography," Microvasc. Res. 80(1), 89-98 (2010).

170. W. Wieser et al., "High definition live 3D-OCT in vivo: design and evaluation of a 4D OCT engine with $1 \mathrm{GVoxel} / \mathrm{s}$, , Biomed. Opt. Express 5(9), 2963-2977 (2014).

171. D.-h. Choi et al., "Spectral domain optical coherence tomography of multi-MHz A-scan rates at $1310 \mathrm{~nm}$ range and real-time 4D-display up to 41 volumes/second," Biomed. Opt. Express 3(12), 3067-3086 (2012).

172. Z. Zhi et al., "4D optical coherence tomography-based micro-angiography achieved by 1.6-MHz FDML swept source," Opt. Lett. 40(8), 1779-1782 (2015).

173. Z. Ding et al., "High-resolution optical coherence tomography over a large depth range with an axicon lens," Opt. Lett. 27(4), 243-245 (2002).

174. R. A. Leitgeb et al., "Extended focus depth for Fourier domain optical coherence microscopy," Opt. Lett. 31(16), 2450-2452 (2006).

175. L. Liu et al., "Binary-phase spatial filter for real-time swept-source optical coherence microscopy," Opt. Lett. 32(16), 2375-2377 (2007).

176. L. Liu et al., "Imaging the subcellular structure of human coronary atherosclerosis using micro-optical coherence tomography," Nat. Med. 17(8), 1010-1014 (2011).

177. C. Blatter et al., "In situ structural and microangiographic assessment of human skin lesions with high-speed OCT," Biomed. Opt. Express 3(10), 2636-2646 (2012).

178. K. Sasaki et al., "Extended depth of focus adaptive optics spectral domain optical coherence tomography," Biomed. Opt. Express 3(10), 2353-2370 (2012).

179. J. Jang et al., "Complex wavefront shaping for optimal depth-selective focusing in optical coherence tomography," Opt. Express 21(3), 28902902 (2013).

180. J. Mo, M. de Groot, and J. F. de Boer, "Focus-extension by depthencoded synthetic aperture in Optical Coherence Tomography," Opt. Express 21(8), 10048-10061 (2013).

181. J. Liang, D. R. Williams, and D. T. Miller, "Supernormal vision and high-resolution retinal imaging through adaptive optics," JOSA A 14(11), 2884-2892 (1997).

182. M. Rueckel, J. A. Mack-Bucher, and W. Denk, "Adaptive wavefront correction in two-photon microscopy using coherence-gated wavefront sensing," Proc. Natl. Acad. Sci. U.S.A. 103(46), 17137-17142 (2006).

183. K. Ferrara, R. Pollard, and M. Borden, "Ultrasound microbubble contrast agents: fundamentals and application to gene and drug delivery," Anпu. Rev. Biomed. Eng. 9, 415-447 (2007).

184. H. Lusic and M. W. Grinstaff, "X-ray-computed tomography contrast agents," Chem. Rev. 113(3), 1641-1666 (2012).
185. A. S. Merbach, L. Helm, and É. Tóth, The Chemistry of Contrast Agents in Medical Magnetic Resonance Imaging, John Wiley \& Sons, New York (2013).

186. J. Chen et al., "Gold nanocages: bioconjugation and their potential use as optical imaging contrast agents," Nano Lett. 5(3), 473-477 (2005).

187. R. John et al., "Targeted multifunctional multimodal protein-shell microspheres as cancer imaging contrast agents," Mol. Imaging Biol. 14(1), 17-24 (2012).

188. T. M. Lee et al., "Engineered microsphere contrast agents for optical coherence tomography," Opt. Lett. 28(17), 1546-1548 (2003).

189. C. Xu et al., "Near-infrared dyes as contrast-enhancing agents for spectroscopic optical coherence tomography," Opt. Lett. 29(14), 1647-1649 (2004).

190. H. Cang et al., "Gold nanocages as contrast agents for spectroscopic optical coherence tomography," Opt. Lett. 30(22), 3048-3050 (2005).

191. A. L. Oldenburg et al., "Imaging gold nanorods in excised human breast carcinoma by spectroscopic optical coherence tomography," J. Mater. Chem. 19(35), 6407-6411 (2009).

192. M. C. Skala et al., "Photothermal optical coherence tomography of epidermal growth factor receptor in live cells using immunotargeted gold nanospheres," Nano Lett. 8(10), 3461-3467 (2008).

193. L. P. Hariri et al., "Endoscopic optical coherence tomography and laser-induced fluorescence spectroscopy in a murine colon cancer model," Laser Surg. Med 38(4), 305-313 (2006).

194. C. A. Patil et al., "Combined Raman spectroscopy and optical coherence tomography device for tissue characterization," Opt. Lett. 33(10), 1135-1137 (2008).

195. J. Wang et al., "Development of a hybrid Raman spectroscopy and optical coherence tomography technique for real-time in vivo tissue measurements," Opt. Lett. 41(13), 3045-3048 (2016).

Jianfeng Wang received his $\mathrm{PhD}$ in biomedical engineering from the National University of Singapore, Singapore, in 2017. He is currently a postdoctoral research associate in the Biophotonics Imaging Laboratory at the University of Illinois at Urbana-Champaign (UIUC), Urbana, Illinois. His research interests include developing and using optical imaging technologies for biomedical studies.

Yang $\mathrm{Xu}$ received his BEng degree in electronic and computer engineering from Hong Kong University of Science and Technology (HKUST) in 2012, and his MS and PhD degrees in electrical and computer engineering from the University of Illinois Urbana-Champaign in 2014 and 2017, respectively. His research interests include computational optical imaging and deep learning.

Stephen A. Boppart is an Abel Bliss professor of engineering in the Departments of Electrical and Computer Engineering, Bioengineering, and Medicine, and a head of the Biophotonics Imaging Laboratory at the Beckman Institute for Advanced Science and Technology. His interests include the development of optical imaging technologies for medical applications, with emphasis on translating these to clinical applications. He is a fellow of SPIE, IEEE, AAAS, OSA, BMES, and AIMBE. 